Aus der ehem. Abteilung Anaesthesiologische Forschung

(Ehem. Leiter: Prof. Dr. med. G Hellige)

im Zentrum Anaesthesiologie, Rettungs- und Intensivmedizin

der Medizinischen Fakultät der Universität Göttingen

\title{
Regionale Lungenventilation bei gesunden Probanden ermittelt mittels elektrischer Impedanztomographie
}

\author{
Inaugural - Dissertation \\ zur Erlangung des Doktorgrades
}

der Medizinischen Fakultät

der Georg-August-Universität zu Göttingen

\author{
vorgelegt von \\ Patrick Braun \\ aus \\ Tübingen
}


Inhaltsverzeichnis

I. Inhaltsverzeichnis

II. Abkürzungen, Abbildungen und Tabellen .....................................................II

II.1 Liste der verwendeten Abkürzungen ................................................II

II.2 Abbildungsverzeichnis.................................................................. IV

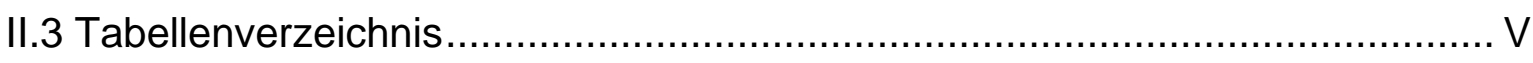

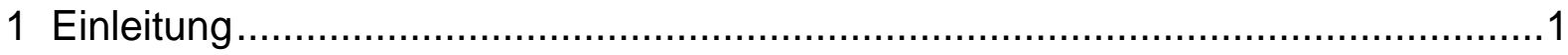

1.1 Die Entdeckung der Atemfunktion...........................................................

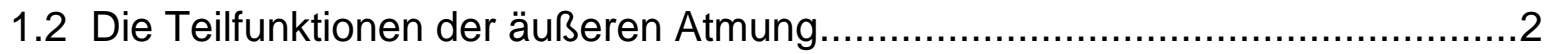

1.3 Die Bedeutung der Gasverteilung ........................................................

1.4 Die Lungenfunktion im Alterungsprozess ..........................................6

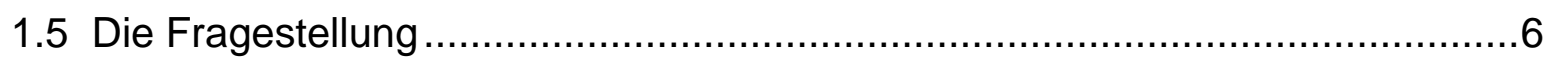

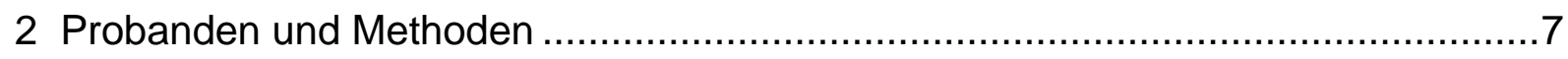

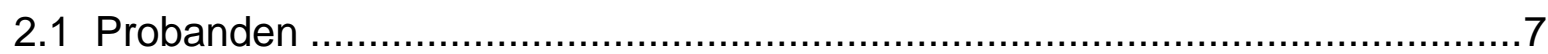

2.2 Untersuchungsverfahren der Lungenfunktion ....................................... 8

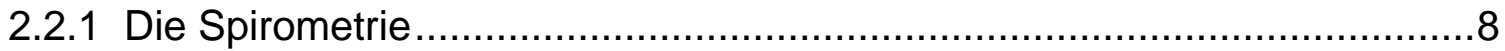

2.2.2 Die Ganzkörperplethysmographie .................................................. 13

2.2.3 Das Messprinzip der Ganzkörperplethysmographie ..............................14

2.2.4 Intrathorakales Gasvolumen und funktionelle Residualkapazität .............17

2.3 Gemessene Lungenfunktionsparameter ........................................... 18

2.4 Die elektrische Impedanztomographie ................................................21

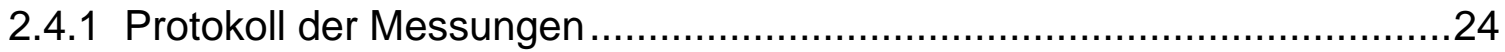

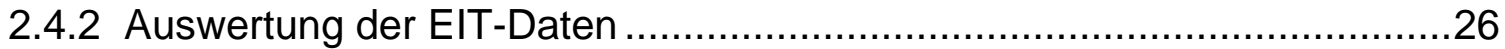

2.4.3 Erstellung von funktionellen EIT-Bildern ..........................................26

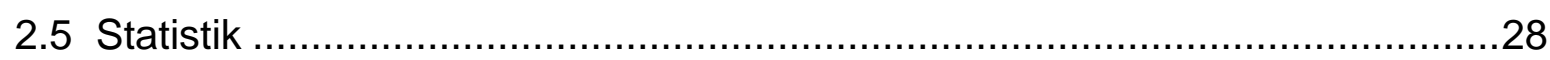

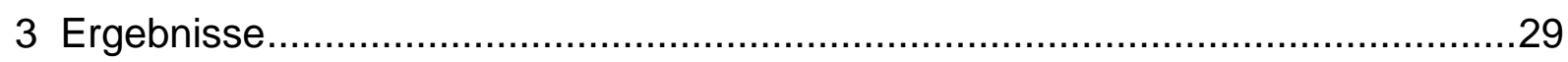

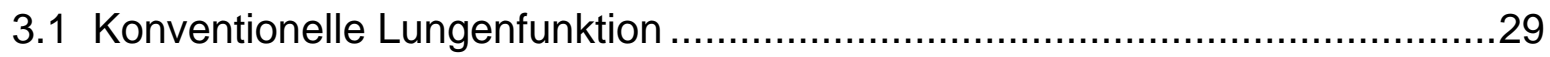

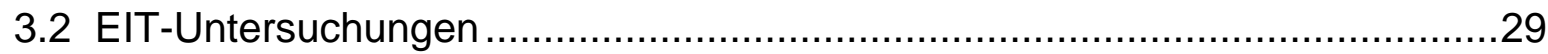

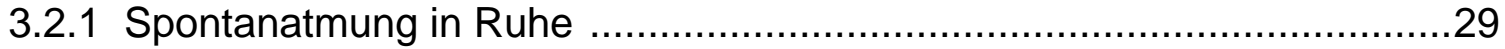

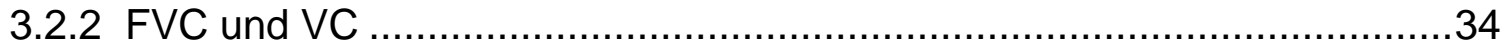

3.2.3 Spontanatmung auf verschiedenen Lungenvolumenniveaus ..................37 


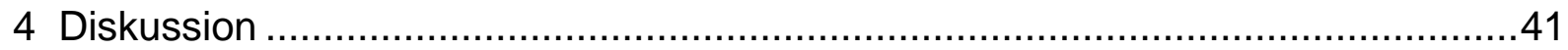

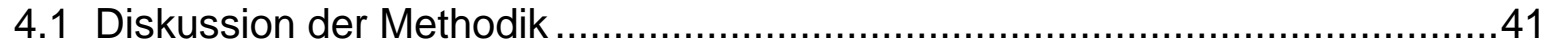

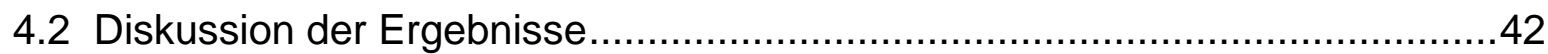

4.2.1 Effekte von Alter und Körperlage .....................................................

4.2.2 Effekte von Alter und Art der Atmung ……...........................................43

4.2.3 Effekte von Alter und Atemgasfluss ......................................................4

4.2.4 Effekte von Alter und Lungenvolumen ..................................................44

4.3 Möglichkeiten der klinischen Anwendung …….........................................

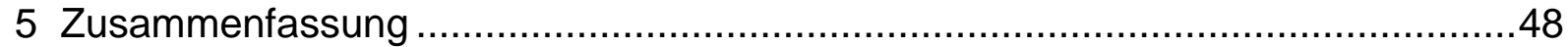

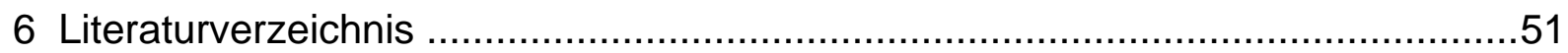




\section{Abkürzungen, Abbildungen und Tabellen}

\section{II.1 Liste der verwendeten Abkürzungen}

CT

Computertomographie

EIT

Elektroimpedanztomographie

ERV

Exspiratorisches Reservevolumen

$\mathrm{FEV}_{1} \quad$ Einsekundenkapazität

FRC Funktionelle Residualkapazität

FVC Forcierte Vitalkapazität

$\mathrm{FVC}_{1} / \mathrm{FVC} \quad$ Tiffenau-Index

IC Inspirationskapazität

IGV Intrathorakales Gasvolumen

IRV Inspiratorisches Reservevolumen

$\mathrm{kPa} \quad$ Kilopascal

MEF Maximaler Exspiratorischer Fluss

MIF Maximaler Inspiratorischer Fluss

MRT Magnetresonanztomographie

$\mathrm{P}_{\mathrm{A}}$

Alveolardruck

PEF Maximale Atemstromstärke

$\mathrm{P}_{\mathrm{K}} \quad$ Kammerdruck

$\mathrm{P}_{\mathrm{N}} \quad$ Druck am Pneumotachographen

$\mathrm{R}$ tot $\quad$ Totraum

RV Residualvolumen

SD Standardabweichung

TLC Totale Lungenkapazität

V Atemstromstärke

$\begin{array}{ll}\mathrm{V}_{\mathrm{T}} & \text { Atemzugvolumen }\end{array}$ 


\section{II.2 Abbildungsverzeichnis}

Abbildung 1: Single-Breath $\mathrm{N}_{2}$-Auswaschverfahren (Einatemzugmethode mit Stickstoff)

Abbildung 2: Einatemzugmethode mit Stickstoff bei einem Patienten mit fortgeschrittener chronischer Bronchitis

Abbildung 3: Funktionsschema eines Pneumotachographen

Abbildung 4: Darstellung der Lungenvolumina und des Tiffenau-Manövers bei einer lungengesunden Person

Abbildung 5: Darstellung der Lungenvolumina und des Tiffenau-Manövers bei einem Patienten mit fortgeschrittener chronischer Bronchitis

Abbildung 6: Flussvolumendiagramm bei einer lungengesunden Person 11

Abbildung 7: Flussvolumendiagramm einer Person mit fortgeschrittener chronischer Bronchitis.

Abbildung 8: Vereinfachte schematische Darstellung der Bodyplethysmographie..

Abbildung 9: Vereinfachte schematische Darstellung der Bodyplethysmographie und Messung der Verschlussdruckkurve bei geschlossenem Atemrohr.

Abbildung 10: Schematische Darstellung des Messprinzips der EIT bei einer EIT-Untersuchung am Thorax.

Abbildung 11: Erzeugung eines funktionellen EIT-Bildes. 
Abbildung 12: Regionale Änderungen des Gasvolumens in der linken und rechten Lungenregion bei einer Gruppe junger und alter Probanden, während normaler Spontanatmung, forcierter Exspiration und langsamer Exspiration und vier verschiedenen Körperpositionen

Abbildung 13: Darstellung des Anteils, den die rechte Lunge an der Gesamtventilation bei den jungen und alten Probanden in der vermessenen Ebene während normaler Spontanatmung, forcierter Exspiration und langsamer Exspiration in vier verschiedenen Körperpositionen einnimmt.

Abbildung 14: Funktionelle elektrische Impedanztomographie der regionalen Lungenventilation eines 25 Jahre alten und eines 73 Jahre alten gesunden Probanden während normaler Spontanatmung im Sitzen, sowie in rechter und linker Seitenlage

Abbildung 15: Graphischer Vergleich der Lungenvolumenveränderung, die zwischen der TLC und der FRC auftritt, mit der Lungenvolumenänderung, die während der FVC- und VC-Manöver auftritt.

Abbildung 16: Lokale relative Impedanzänderung in der rechten und linken Lungenregion bei einem spontan atmenden 74 Jahre alten Probanden in vier verschiedenen Körperpositionen.

Abbildung 17: Darstellung des Anteils, den die rechte Lunge an der Gesamtventilation in der vermessenen Ebene bei den jungen und alten Probanden, während normaler Spontanatmung und während der Atmung mit einem geringen bzw. hohen Lungenvolumen in vier verschiedene Körperpositionen einnimmt 


\section{II.3 Tabellenverzeichnis}

Tabelle 1: Biometrische Daten und Ergebnisse der

Lungenfunktionsuntersuchung aller Probanden.............................. 7

Tabelle 2: Elektrische Impedanzen biologischer Gewebe.................................. 21 


\section{Einleitung}

\subsection{Die Entdeckung der Atemfunktion}

Es ist kein Zufall, dass die Entdeckung des Blutkreislaufes durch William Harvey im Jahre 1628 wesentlich eher erfolgte, als dies bei der Atmung möglich gewesen wäre. Harvey, der die Kreislauffunktion als Bewegung des Herzens und des Blutes bezeichnete, entwickelte seine Theorie aus Sektionen von Leichen und physiologischen Tierexperimenten (Lyons und Keiner 1980). Er zeigte, dass Blut wegen der Klappen im Herzen und in den Venen nur in eine Richtung fließen kann und stellte fest, dass das Herz eines Tieres sich nach der Entfernung wie ein Muskel weiter dehnte und zusammenzog. Damit ist der Kreislauf, für den der Begriff "Zirkulation“ von einem Vorgänger Harveys, Andrea Cesalpino, geprägt worden war, ein im Körper geschlossenes System, dessen Entdeckung mit den Kenntnissen der Zeit möglich war.

Das Wesen der Atmung war der wissenschaftlichen Erkenntnis erst mehr als 160 Jahre später zugänglich, als Antoine Laurent de Lavoisier im Jahre 1783 seine erste Betrachtung zur Atmung der Tiere anstellte (Seguin und Lavoisier 1783). Zu diesem Zeitpunkt beherrschte die elegante, aber spiegelbildlich falsche Phlogiston-Theorie der Verbrennung und Atmung das wissenschaftliche Denken, und die Zusammensetzung der ein- und ausgeatmeten Luft war nicht bekannt. Lavoisier ging von der Beobachtung aus, dass Metalle bei der Kalzination (Oxydierung) an Gewicht zunehmen. Er beschrieb diesen Vorgang als Anlagerung von Luft und erkannte damit auch, dass Luft aus zwei verschiedenen elastischen Flüssigkeiten (Gasen) zusammengesetzt ist, atembar und nicht atembar. Nach Experimenten mit einem Sperling, der in einem geschlossenen Gefäß atmete, bis er verendete, erklärte er die Atmung als einen Vorgang, bei dem atembare Luft (Sauerstoff) in fixe Luft (Kohlendioxyd) umgewandelt wird, wobei der inerte Teil der Luft (Stickstoff) nicht an der Atmung teilnimmt. Neben vielen anderen herausragenden Leistungen benannte er damit die atembare Luft als Sauerstoff, erklärte das Wesen von Atmung und Verbrennung und widerlegte die Phlogistontheorie, der zufolge entzündliche Materie bei der Verbrennung eine besondere Substanz an die Luft abgeben soll (Braun 1988). Damit ist die Atmung im Gegensatz zum Kreislauf ein offenes System mit Stoffaustausch zur Umgebung hin, das man nur verstehen kann, wenn die Art der am Austausch beteiligten Gase bekannt ist. 


\subsection{Die Teilfunktionen der äußeren Atmung}

Weitere differenzierende Untersuchungen zum Prozess der äußeren Atmung haben gezeigt, dass sich diese aus vier hintereinandergeschalteten Teilvorgängen zusammensetzt, Ventilation, Gasverteilung, Diffusion und Lungenperfusion. Die Ventilation entspricht dem durch Muskelkraft verursachten konvektiven Ein- und Auswärtstransport von Luft und Exspirationsgas. Die Gasverteilung erfolgt mit dem Ziel, Gas in Richtung zu und von den Alveolen zu bewegen. Die Diffusion ist der durch den Konzentrationsgradienten bedingte physikalische Gastransfer an den Lungen- und Blutgefäßmembranen. Die Perfusion ist die zum Gasaustausch erforderliche Lungendurchblutung.

\subsection{Die Bedeutung der Gasverteilung}

Die Gasverteilung wird als Prozess anschaulicher, wenn wir uns die Lunge in Richtung auf die Austauschmembranen als Trompete vorstellen, da zur Peripherie hin der Durchmesser der Atemwege größer und die Geschwindigkeit der Gasbewegung langsamer wird. Unmittelbar vor der Alveolarmembran ist sie so langsam, dass der konvektive Transport durch Diffusion in der Gasphase ergänzt wird. Die Bedeutung der Gasverteilung für den Gasaustauschprozess konnten erstmals Comroe (Comroe und Fowler 1951) im single-breath StickstoffauswaschVerfahren belegen. Dabei wird aus normaler Ruheatmung langsam maximal ausgeatmet und anschließend bis zur vollständigen Lungenfüllung Sauerstoff eingeatmet. Daraufhin erfolgt die vollständige Ausatmung. Das beschriebene singlebreath Stickstoffauswasch-Verfahren ist in Abbildung 1 dargestellt. 


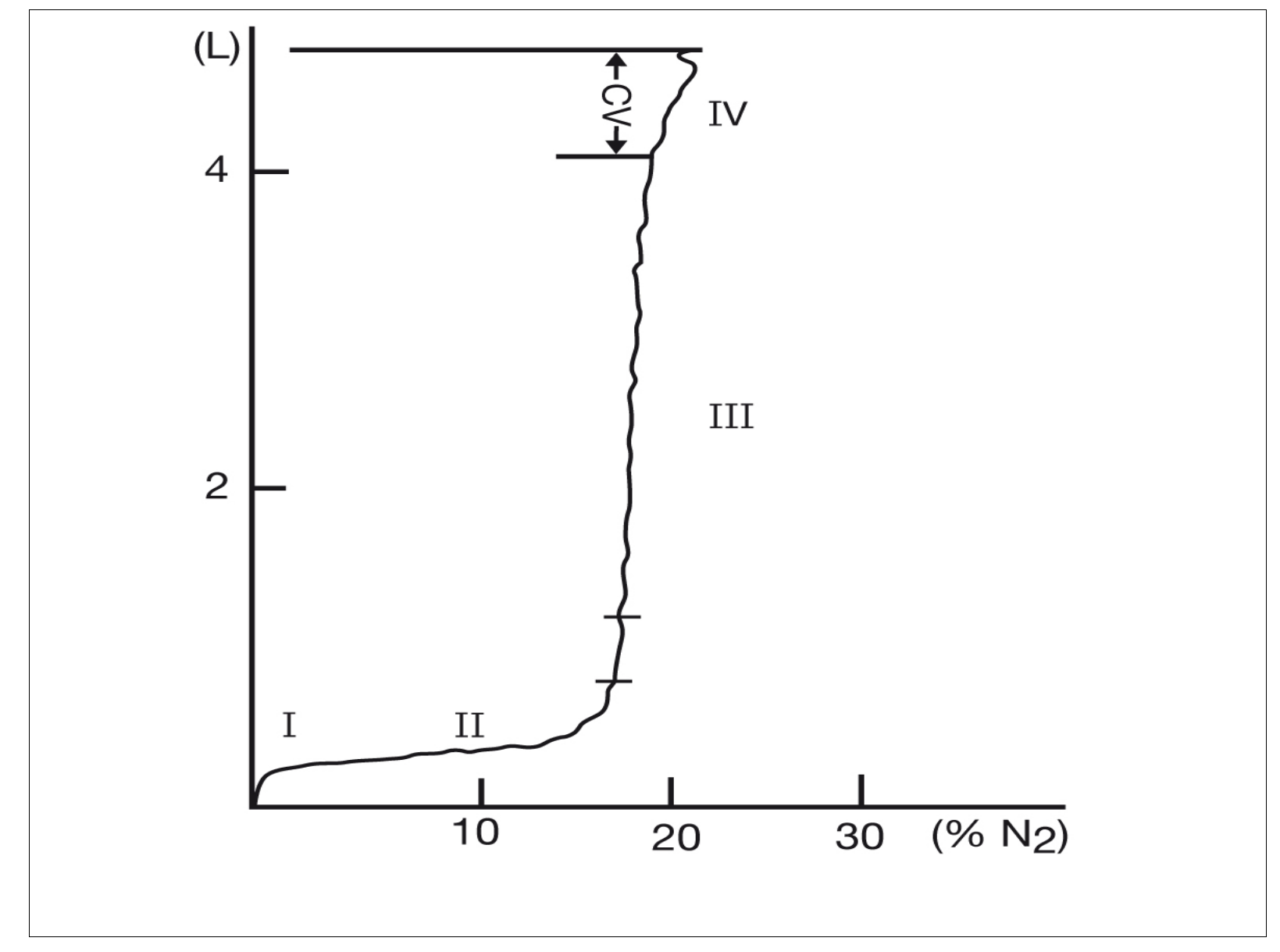

\section{Abbildung 1:}

Single-Breath $\mathrm{N}_{2}$-Auswaschverfahren (Einatemzugmethode mit Stickstoff). Dargestellt ist die Stickstoffkonzentration (Abszisse) gegen das Volumen (Ordinate). Die Phasen der Ausatmung sind: I Totraum, II Mischluft, III alveoläres Plateau, IV Closing Volume (Braun und Voigt 1978, S.165)

Bei dieser Methode wird die Stickstoffkonzentration der Ausatemluft mit einem schnellen Stickstoffanalysator bestimmt. Zunächst wird reiner Sauerstoff abgegeben, der dem anatomischen Totraum entspricht (Phase I).

Bei der weiteren Ausatmung entweicht Mischluft (Phase II), gefolgt von der homogenen Alveolarluft (Phase III). Die Phase III wird auch als Plateau-Phase bezeichnet.

Die relativ gleichförmige Stickstoff-Konzentration der Plateau-Phase ergibt sich dadurch, dass eine große Zahl von Alveolen mit etwa gleicher Geschwindigkeit bzw. gleichen Zeitkonstanten belüftet wird. 
Der Anstieg der $\mathrm{N}_{2}$-Konzentration zwischen 750 und $1250 \mathrm{ml}$ der Ausatmung liegt daher bei der gesunden Lunge unter 1,5\% $\Delta \mathrm{N}_{2}$ (Comroe und Fowler 1951). Nach Ende der Phase III kommt es zu einem stärkeren Anstieg der StickstoffKonzentration (Phase IV). Das Gas dieser letzten Phase der Ausatmung stammt aus Alveolarbereichen, die zu Beginn der Sauerstoff-Inspiration noch geschlossen waren und sich erst während der Inspiration geöffnet haben. Man spricht daher von ClosingVolume. Die Belüftung der Alveolen erfolgt hier langsamer, mit anderen Zeitkonstanten, und die Stickstoff-Konzentration ist höher. Bei einer fortgeschrittenen chronisch-obstruktiven Lungenerkrankung bildet sich im single-breath $\mathrm{N}_{2}$ Auswaschverfahren kein Plateau Ein Closing-Volume ist nicht abgrenzbar. (Abbildung 2)

Es gibt keine homogene Alveolarluft. Die einzelnen Alveolarbezirke werden hintereinander, mit unterschiedlichen Zeitkonstanten belüftet. Der $\Delta \mathrm{N}_{2}$-Wert liegt meist deutlich über 1,5\% (Braun und Voigt 1978). Er ist in den Abbildungen 1 und 2 zwischen den waagerechten Linien markiert. Ventilatorische Verteilungsstörungen treten regional auf, die Stickstoff- und Fremdgas-Auswaschverfahren können sie jedoch nur global erfassen.

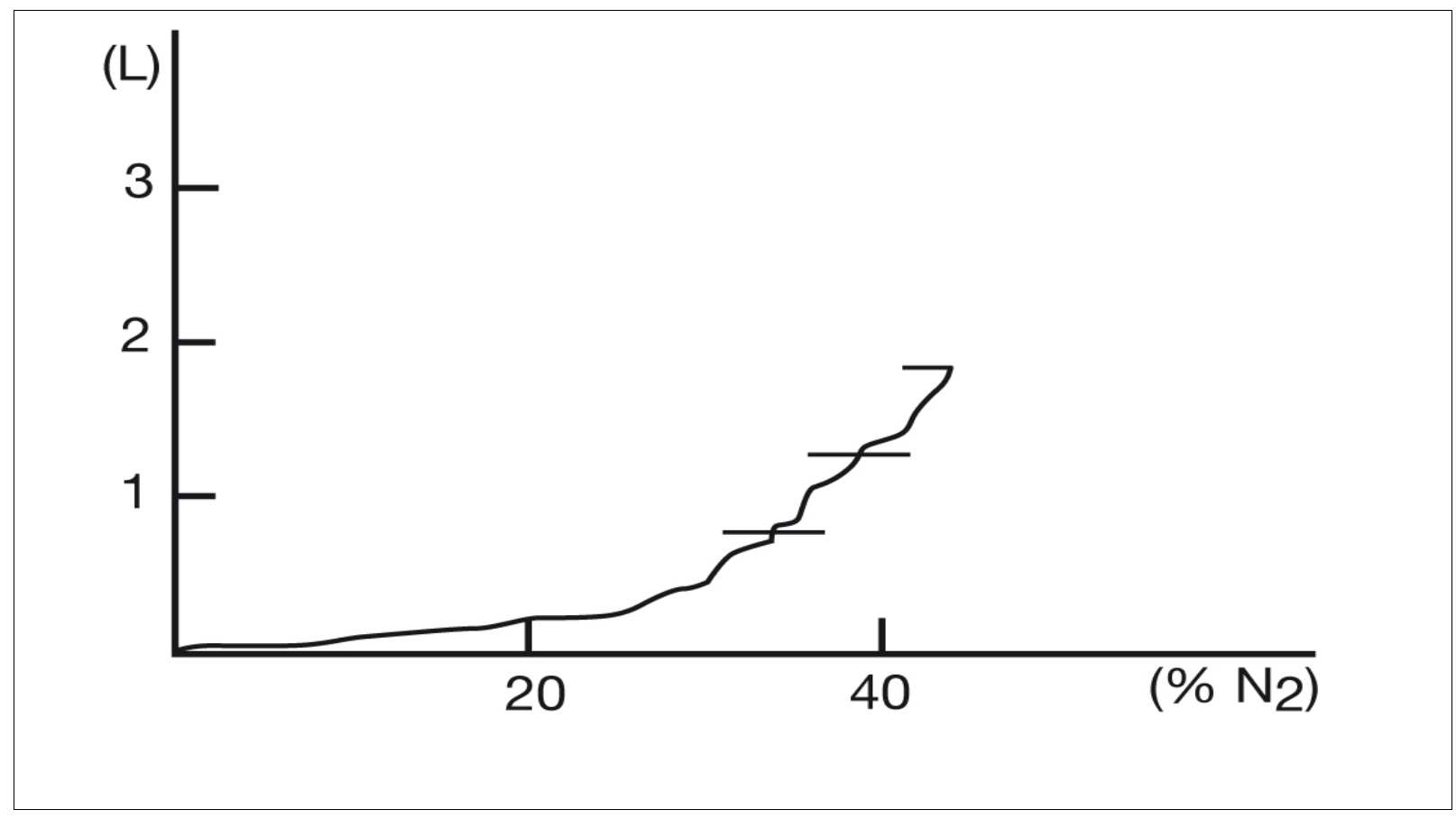

Abbildung 2

Einatemzugmethode mit Stickstoff bei einem Patienten mit fortgeschrittener chronischer Bronchitis. Eine Phase III mit einer relativ homogenen Alveolarluft existiert nicht, die Stickstoffkonzentration steigt stark an. $\Delta N_{2}$ ist mit 5,3\% stark pathologisch verändert. (Braun und Voigt 1978, S.168) 
Bei der gesunden Lunge besteht durch das Eigengewicht des Organs eine Differenz des negativen Pleuradruckes von etwa $7,5 \mathrm{~cm} \mathrm{H}_{2} \mathrm{O}$ zwischen den apikalen und basalen Pleurabezirken (Bates et al. 1971). In der Atemmittellage, bei der funktionellen Residualkapazität (FRC), betragen die absoluten Werte oben in der Pleuraspitze $-10 \mathrm{~cm} \mathrm{H}_{2} \mathrm{O}$ und unten oberhalb des Zwerchfells $-2,5 \mathrm{~cm} \mathrm{H} \mathrm{H}_{2} \mathrm{O}$. Nach maximaler Einatmung, bei der totalen Lungenkapazität (TLC) liegen die Werte bei 40 bzw. $-32,5 \mathrm{~cm} \mathrm{H}_{2} \mathrm{O}$. Nach maximaler Ausatmung, beim Residualvolumen (RV), finden sich Werte von $-4 \mathrm{~cm}$ (oben) und $+2,5 \mathrm{~cm}$ (unten). Damit erklärt sich die Lokalisation des als Closing Volume (Phase IV) bezeichneten Volumens bei der gesunden Lunge, das aus den ganz basalen Lungenpartien stammt, die sich erst während der Inspiration öffnen. Bei ruhiger Atmung in Atemmittellage werden die etwas höher gelegenen basalen Lungenpartien besser ventiliert als die apikalen und der Hauptanteil der homogenen Gasverteilung (Phase III, Plateauphase) stammt aus diesen basalen Lungenbezirken.

Bei Adipositas, bei chronischen Lungenkrankheiten, bei Alterungsprozessen und auch bei Elastizitätsverlust der Lungen aus anderen Gründen kann das Closing Volume nach oben in den Bereich der Atemmittellage wandern, so dass auch der Gasaustausch der Atemmittellage, d.h. die normale Ruheatmung, durch passageren regionalen Atemwegsverschluss beeinträchtigt ist. Die Abhängigkeit des Gasaustausches vom Eigengewicht der Lunge ist unabhängig von der Körperlage und tritt auch in Rücken- oder Bauchlage auf. Die beschriebenen Veränderungen ergeben sich aus dem Verlauf der Ruhedehnungskurve der Lunge. Sie erleichtern die Zuordnung der Ventilation zur Perfusion, da auch bei letzterer eine Abhängigkeit von der Schwerkraft besteht.

Aus den oben dargestellten Zusammenhängen ergibt sich, dass die Gasverteilung eine besondere Bedeutung bei respiratorischen Krankheitsprozessen hat und dass ihre Erfassung mit konventionellen Funktionsanalysen wichtige Teilaspekte wie regionale Ausprägung nicht erkennen kann. Therapeutische Überlegungen müssen jedoch regionale Aspekte von Funktionsstörungen der Lunge mit in Betracht ziehen. Dies gilt umso mehr, als in der Intensivmedizin unter der Beatmungstherapie pulmonale Erkrankungen beobachtet werden, die es ohne Intensivmedizin nicht gegeben hat, wie z.B. die Beatmungspneumonie. Hier werden neue methodische Ansätze benötigt, die die regionale Gasverteilung bettseitig erfassen können und mit vertretbarem Aufwand diese Information für die Diagnostik und Therapie nutzen können. 


\subsection{Die Lungenfunktion im Alterungsprozess}

Viele im Rahmen der Intensivmedizin behandelten Patienten befinden sich im höheren Lebensalter. Versteifung der Thoraxwand, Verlust der Lungenelastizität und eine verminderte Kraft der respiratorischen Muskulatur entsprechen altersbedingten Veränderungen der Atemmechanik (Gibson et al. 1976, Rossi et al. 1996, Babb und Rodarte 2000, Stenqvist 2003). Dabei ist der Alterungsprozess der Lunge mit globalen statischen und dynamischen Lungenfunktionsparametern gut untersucht (Campbell und Lefrak 1978, Rossi et al. 1996, Zeleznik 2003). Die Gasverteilung im Alter ist wenig untersucht, außer bei einigen mit radioaktiven Gasen durchgeführten Studien zur topographischen Gasverteilung bei langsamen Atemmanövern (Holland et al. 1968, Kronenberg et al. 1973). Diese Methoden erlauben jedoch keine kontinuierliche Anwendung.

\subsection{Die Fragestellung}

Eine neuartige, in Entwicklung befindliche strahlungsfreie, bildgebende Methode ist die elektrische Impedanztomographie (EIT), die mit geringer Invasivität thorakale Schnittbilder erzeugen kann, die kontinuierlich erfassbar sind und über die regionale Gasverteilung Auskunft geben. Die Methode ist heute noch nicht im gewünschten Sinne klinisch einsetzbar, erlaubt aber schon die Anwendung für einige grundsätzliche Fragestellungen, die die Möglichkeiten und Grenzen des Verfahrens aufzeigen.

Eine grundsätzliche Fragestellung ist der Einfluss des Alters auf die Gasverteilung der Lunge. Es ist das Ziel dieser Studie, die moderne EIT-Technologie einzusetzen, um die altersbedingten Veränderungen der regionalen Gasverteilung im Verhältnis zu den Bedingungen bei jungen Erwachsenen zu erfassen. Dabei soll auch geprüft werden, wie Lageveränderungen und spezielle Atemmanöver die Gasverteilung beeinflussen. 


\section{Probanden und Methoden}

\subsection{Probanden}

In dieser Studie wurde eine Gruppe von 8 jungen (26 \pm 1 Jahre, \pm SD) und 8 älteren (75 \pm 5 Jahre, \pm SD) gesunden Männern mittels EIT (Tomograph Goe-MF II, Univ. of Göttingen, Germany) untersucht.

Die Studie war von der zuständigen Ethikkommission genehmigt. Alle Probanden haben nach einem Aufklärungsgespräch schriftlich ihre Mitarbeit dokumentiert. Untersucht wurden ausschließlich Probanden, die bis zum Zeitpunkt der Studie Nichtraucher waren und weder anamnestisch noch im Rahmen einer körperlichen Untersuchung Anzeichen einer Vorerkrankung von Lunge, oder HerzKreislaufsystem aufwiesen. Die Lungenfunktion der untersuchten Gruppe wurde spirometrisch und mittels Ganzkörperplethysmographie (Masterlab, Jaeger, Würzburg, FRG) bestimmt. Zur Beurteilung der Lungenfunktion wurden die gemessenen Werte mit den empfohlenen Referenzwerten der European Respiratory Society (Quanjer et al. 1993) verglichen. Einen Überblick über Alter, Körpergröße und Gewicht gibt Tabelle 1.

\begin{tabular}{|c|c|c|c|c|c|c|c|c|c|c|c|c|c|c|c|c|c|c|c|}
\hline Proband & Alter & Gewicht $(\mathrm{kg})$ & Grösse (cm) & FEV 1 & \%pred & PEF & \%pred & FVC & \%pred & FRC & \%pred & RV & \%pred & TLC & \%pred & $\mathrm{R}$ tot & \%pred & FEV1/FV & \%pred \\
\hline 01 & 25 & \begin{tabular}{|r|}
72 \\
\end{tabular} & 176 & 4,16 & 95,7 & $\overline{9,23}$ & 93,4 & 4,86 & 94,3 & 2,73 & 83,8 & 1,13 & 69,2 & $\overline{5,98}$ & 85,7 & 0,73 & 244,5 & $0,0,86$ & $\overline{~ 104,2}$ \\
\hline 02 & 26 & 78 & 186 & 5,61 & 117,3 & 12,57 & 119,8 & 7,18 & 125,4 & 4,74 & 136 & 1,88 & 107,2 & 9,06 & 116,4 & 0,12 & 38,6 & 0,78 & 96,9 \\
\hline 03 & 26 & 94 & 194 & 5,46 & 107,2 & 9,58 & 87,5 & 6,58 & 106,8 & 4,47 & 121,3 & 2,11 & 112 & 8,69 & 103,1 & 0,18 & 60,6 & 0,83 & 101,6 \\
\hline 104 & 24 & 86 & 188 & 5,34 & 109,7 & 8,59 & 80,9 & 6,78 & 116,1 & 4,07 & 115,2 & 1,52 & 85,4 & 8,3 & 104,5 & 0,44 & 147,3 & 0,79 & 98,5 \\
\hline 05 & 25 & 90 & 193 & 4,81 & 94,6 & 10,57 & 96,8 & 5,95 & 97,1 & 4,4 & 120,6 & 1,79 & 97 & 7,75 & 92,9 & 0,13 & 44,2 & 0,81 & 105,2 \\
\hline 106 & 26 & 70 & 186 & 4,79 & 100,8 & 9,6 & 91,9 & 5,58 & 98 & 3,66 & 104,7 & 1,29 & 72,4 & 6,87 & 88,3 & 0,17 & 56,1 & 0,86 & 107,6 \\
\hline 509 & 26 & 93 & 196 & 5,09 & 98,3 & 10,37 & 93,7 & 7,16 & 114,1 & 4,22 & 113 & 2,03 & 106,1 & 9,19 & 107,1 & 0,31 & 104,3 & 0,71 & 88,5 \\
\hline 11 & 26 & 89 & 184 & 4,41 & 103 & 8,14 & 99,5 & 5,9 & 110,7 & 1,89 & 54,8 & 1,15 & 65,3 & 5,82 & 96,1 & 0,65 & 83,5 & 0,75 & 98,8 \\
\hline Mittelwert & 26 & 84 & 188 & 4,96 & 103,3 & 9,83 & 95,4 & 6,25 & 107,8 & 3,77 & 106,2 & 1,61 & 89,3 & 7,71 & 99,3 & 0,34 & 97,4 & 0,80 & 100,2 \\
\hline SD & & 10 & 7 & 0,51 & 7,7 & 1,37 & 11,4 & 0,82 & 10,8 & 0,98 & 25,6 & 0,34 & 16,9 & 1,15 & 8,8 & 0,18 & 36,6 & 0,05 & 5,8 \\
\hline
\end{tabular}

\begin{tabular}{|c|c|c|c|c|c|c|c|c|c|c|c|c|c|c|c|c|c|c|c|}
\hline Proband & Alter $1 \mathrm{C}$ & Gewicht (kg) & Grosse (cm) & FEV T & \%pred & PEF & \%pred & $\mathrm{FVC}$ & \%pred & FRC & \%pred & RV & \%pred & TLC & \%pred & $\mathrm{R}$ tot & \%pred & $\mathrm{FEV} 1 / \mathrm{FV}$ & \%pred \\
\hline a01 & 76 & 105 & 186 & 3,26 & 96,9 & 7,66 & 91,3 & 4,27 & 96 & $\overline{4,37}$ & 1111,3 & $\overline{3,47}$ & 122,5 & $\overline{7,74}$ & 99,5 & 0,32 & $\overline{107,4}$ & 0,76 & 105,5 \\
\hline 202 & 77 & 86 & 173 & 2,94 & 106 & 5,61 & 74,3 & 3,89 & 105,7 & 3,8 & 104,5 & 2,69 & 100,2 & 6,58 & 97,6 & 0,22 & 74,2 & 0,76 & 104,2 \\
\hline 203 & 73 & 79 & 165 & 2,58 & 102,5 & 4,77 & 66,4 & 3,97 & 120,5 & 4,91 & 143,6 & 3,94 & 146,4 & 7,9 & 129,5 & 0,4 & 133 & 0,65 & 90,9 \\
\hline 204 & 61 & 85 & 172 & 2,7 & 86 & 6,77 & 83,7 & 3,05 & 76,6 & 2,86 & 82 & 1,76 & 74,2 & 4,81 & 72,1 & 0,18 & 61,4 & 0,89 & 120,3 \\
\hline 205 & 76 & 70 & 172 & 2,92 & 107,1 & 8,3 & 110,8 & 3,53 & 97,6 & 3,84 & 106,5 & 3,3 & 123,4 & 6,83 & 102,5 & 0,15 & 51 & 0,83 & 141,3 \\
\hline 206 & 77 & 83 & 178 & 2,45 & 81,9 & 3,78 & 48,2 & 3,27 & 82,5 & 4,19 & 111,6 & 2,82 & 102,4 & 6,09 & 85,3 & 0,26 & 87,9 & 0,75 & 108,3 \\
\hline 209 & 72 & 74 & 172 & 2,45 & 86 & 7,05 & 92 & 3,06 & 82,3 & 3,31 & 92,6 & 2,43 & 94,1 & 5,5 & 82,5 & 0,3 & 99,2 & 0,80 & 115,1 \\
\hline 910 & 73 & 73 & 171 & 3,48 & 106 & 6,69 & 93,7 & 4,26 & 100 & 3,03 & 85,5 & 2,58 & 102,2 & 6,84 & 96,1 & 0,2 & 91,8 & 0,82 & 108,6 \\
\hline Mittelwert & 73 & 82 & 174 & 2,85 & 96,6 & 6,33 & 82,6 & 3,66 & 95,2 & 3,79 & 104,7 & 2,87 & 108,2 & 6,54 & 95,6 & 0,25 & 88,2 & 0,78 & 111,8 \\
\hline SD & 5 & 6 & 4 & 0,34 & 10,4 & 1,41 & 18,9 & 0,44 & 14,4 & 0,70 & 19,4 & 0,64 & 21,2 & 0,93 & 17,0 & 0,08 & 25,1 & 0,07 & 14,5 \\
\hline
\end{tabular}

Tabelle 1:

Biometrische Daten und Ergebnisse der Lungenfunktionsuntersuchung aller Probanden. 


\subsection{Untersuchungsverfahren der Lungenfunktion}

\subsubsection{Die Spirometrie}

Die üblicherweise als Lungenfunktion bezeichneten Untersuchungsverfahren befassen sich weniger mit dem Gasaustausch als mehr mit der Durchgängigkeit des bronchialen Systems und der Bestimmung verschiedener Lungenvolumina.

Die am häufigsten durchgeführte Lungenfunktionsuntersuchnung ist die Spirometrie (Ulmer et al. 1991). Bei dieser Untersuchung wird mit einem elektronischen Flusssensor der beim Atmen entstehende Gasstrom erfasst. Ein solcher Flusssensor wurde im Jahre 1925 von Fleisch entwickelt (Fleisch 1925). Mit Hilfe dieses Pneumotachographen ist es möglich, den beim Atmen entstehenden Luftstrom zu messen. Das Messprinzip beruht auf dem Gesetz von Hagen-Poiseuille, nach dem die Strömungsgeschwindigkeit in einem starren Rohr bei laminarer Strömung proportional zur Druckdifferenz pro Längeneinheit ist. Um die Luftströmung in einem solchen Atemrohr laminar zu halten, wurde ein System vieler kleiner paralleler Röhren verwendet. Heute werden anstatt paralleler Röhren beheizte feinmaschige Siebe verwendet, deren Widerstand so klein gehalten ist, dass sie Ein- und Ausatemstrom nicht beeinflussen.

Durch Drucksensoren wird die Druckdifferenz direkt vor und hinter dem Sieb gemessen. Die Druckdifferenz, die zwischen beiden Sensoren gemessen wird, ist der jeweilig herrschenden Strömungsgeschwindigkeit direkt proportional und erlaubt eine Eichung des Gerätes in $\mathrm{ml} / \mathrm{s}$ oder l/s, bzw. nach Integration der Flusskurve in $\mathrm{ml}$ oder I. (Abbildung 3)

Damit kann der Flusssensor oder Pneumotachograph alle atembaren Lungenvolumina, aber auch alle bei den verschiedenen Atemmanövern zugänglichen Flussparameter erfassen (Abbildung 4). 


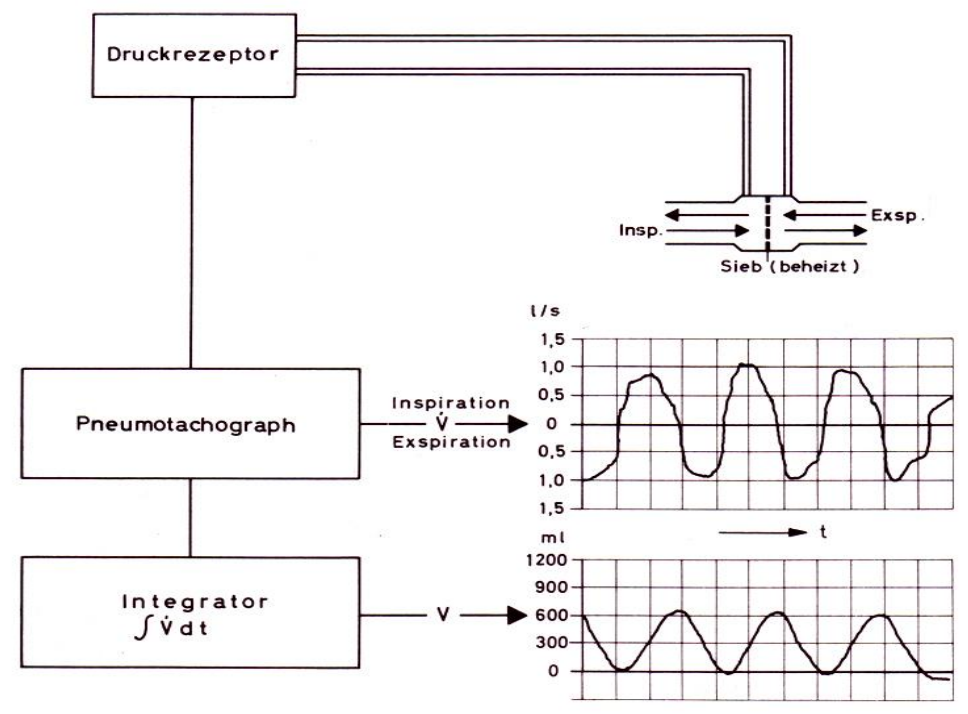

Abbildung 3:

Funktionsschema eines Pneumotachographen. Die in dem Atemrohr vor und hinter einem beheizten Sieb gemessene Druckdifferenz enspricht dem Atemgasstrom, der durch Integration in ein Volumensignal umgewandelt wird.

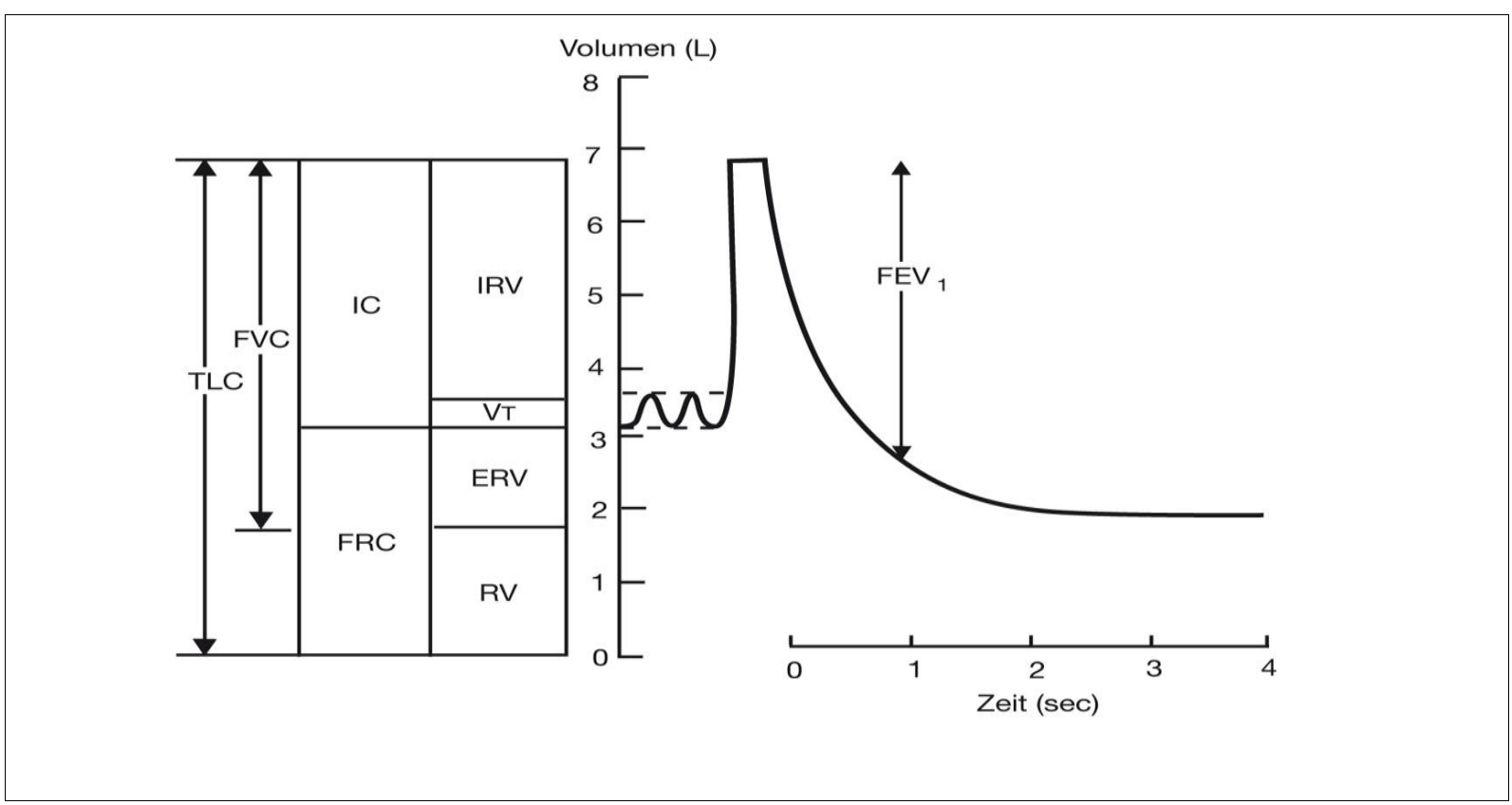

Abbildung 4:

Darstellung der Lungenvolumina und des Tiffenau-Manövers bei einer lungengesunden Person. Die Größe des Tidalvolumens und der Reservevolumina sind links schematisch aufgezeichnet. Die rechts erkennbare rasche Ausatmung nach tiefer Einatmung lässt die Geschwindigkeit der Volumenbewegung erkennen und die Einsekundenkapazität $\left(\mathrm{FEV}_{1}\right)$. 
Nach der spirometrischen Messung der Ruheatmung ( $T V=$ tidal volume) erfolgt die tiefe Ein- und Ausatmung, so dass das inspiratorische (IRV) und exspiratorische Reservevolumen (ERV) zugänglich werden. Das gesamte atembare Volumen, die Vitalkapazität, ist die Summe aus IRV, TV und ERV. Die VC kann durch langsame oder schnelle Atemmanöver erfasst werden. Das Wort Kapazität wird immer dann eingesetzt, wenn zusammengesetzte Volumina bezeichnet werden. Zur Bestimmung der forcierten Vitalkapazität $(F V C)$ und der Einsekundenkapazität $\left(F E V_{1}\right)$ wird der Patient aufgefordert, tief einzuatmen, die Luft kurz anzuhalten und dann kräftig auszuatmen, nachdem die Registriergeschwindigkeit erhöht wurde (Abbildung 4). Die Abbildung zeigt das Spirogramm und die entsprechenden Parameter für einen Lungengesunden.

Bei einem Patienten mit einer krankheitsbedingten Ausatembehinderung wie bei fortgeschrittener chronischer Bronchitis (COLD = chronic obstructive lung disease) erfolgt die forcierte Ausatmung verzögert und der Krankheitsprozess hat zu einer Einschränkung der VC geführt (Abbildung 5).

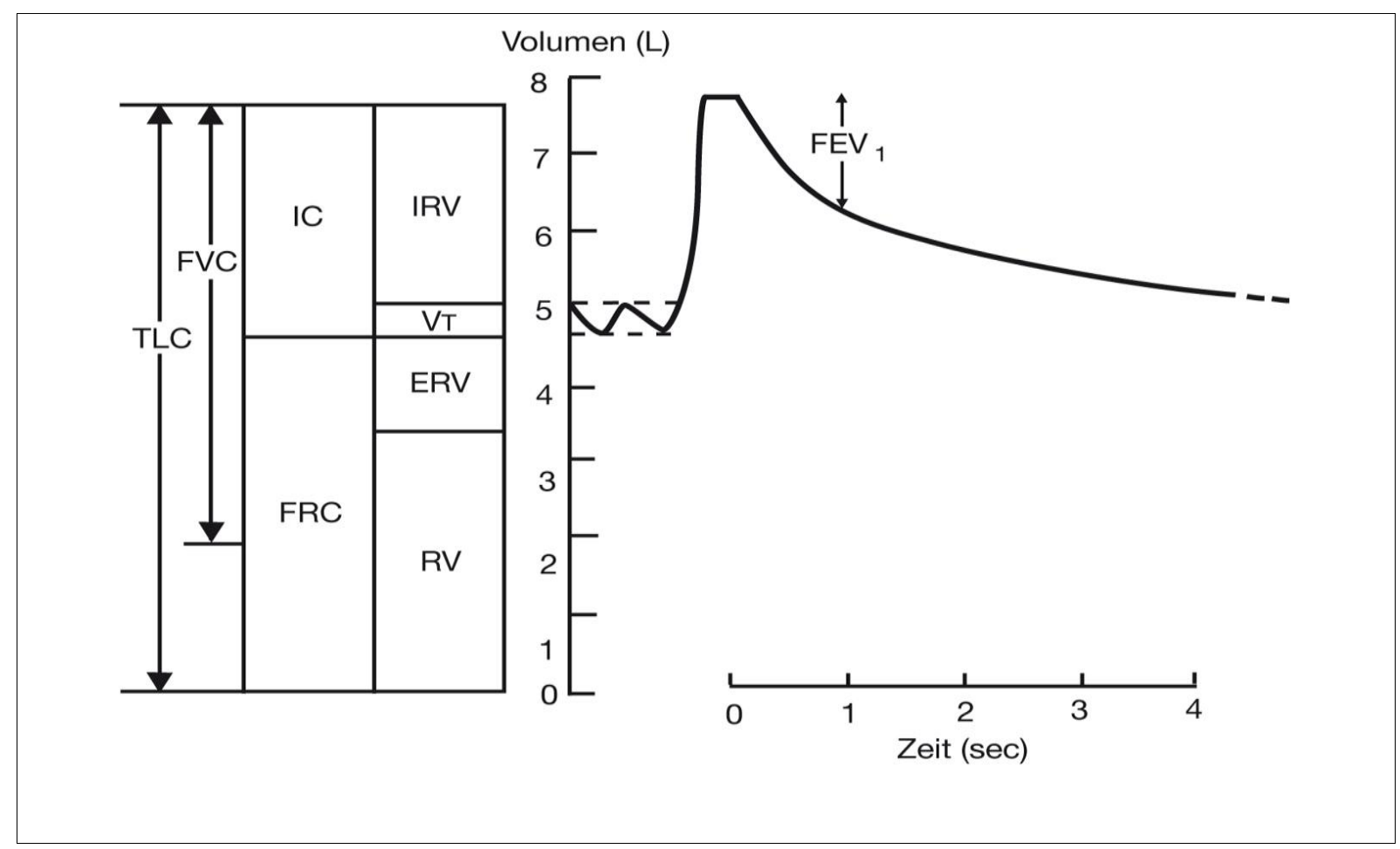

Abbildung 5:

Gleiche Methode wie in Abb 4 bei einem Patienten mit fortgeschrittener chronischer Bronchitis. Die Geschwindigkeit der raschen Ausatmung ist deutlich verzögert und der patientenbezogene FEV -Wert $_{1}$ herabgesetzt, ebenso wie die FVC. Der Krankeitsprozess hat zu einer Lungenblähung geführt, bei der RV und FRC gegenüber der Norm deutlich erhöht sind. 
Da der Flusssensor sowohl den Gasfluss als auch die Volumina erfassen kann, ist die gleichzeitige graphische Darstellung von Fluss und Volumen möglich. Man spricht vom Flussvolumendiagramm (Abbildung 6). Das Flussvolumendiagramm zeigt die Volumina und Flussgrößen in Ruhe und bei maximaler Ein- und Ausatmung. Damit ist die verfügbare Atemreserve als Fläche unmittelbar optisch einsehbar.

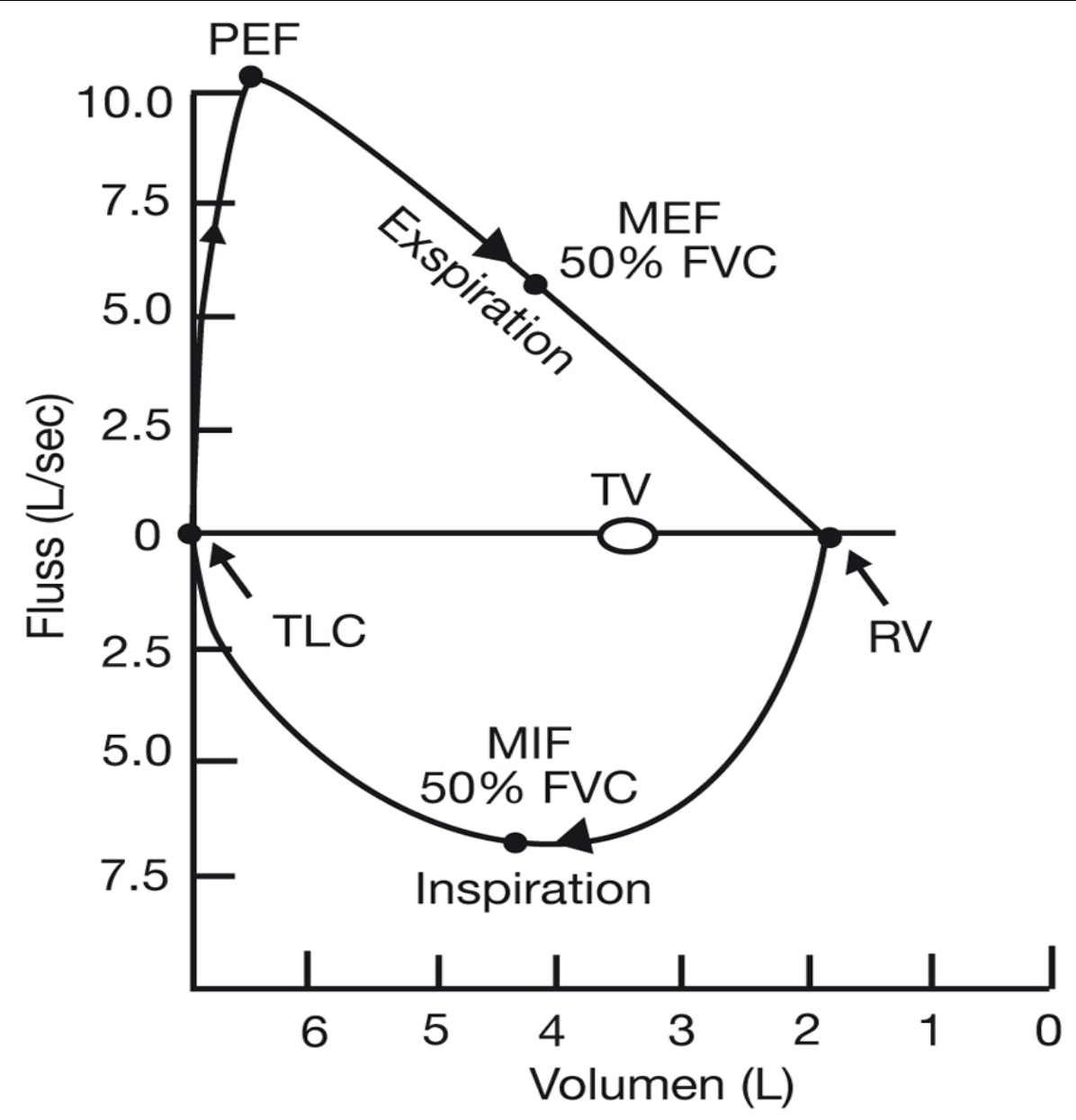

Abbildung 6:

Flussvolumendiagramm bei einer lungengesunden Person. Die Abbildung enthält die Ruheatmung und die maximale Ein- und Ausatmung in einer Darstellung Volumen gegen Atemgasfluss. Die Fläche zwischen dem Tidalvolumen (TV) und der maximalen Ein- und Ausatmung entspricht der pulmonalen Leistungsreserve. Die maximale Einatmung hat eine symmetrische Form, die Ausatmung nicht. MEF 50\% FVC und MIF 50\% FVC bezeichnen die maximale ex- und inspiratorische Stromstärke nach 50\% der maximalen Aus- bzw. Einatmung. Es handelt sich um wichtige atemdynamische Funktionsgrößen im Spirogramm. 
Die rasche Ausatmung nach tiefer Inspiration, das sogenannte Tiffenau-Manöver, erfolgt zunächst mit dem Willen, der Kraft und der Motivation der untersuchten Person. Je weiter die Exspiration fortschreitet, desto mehr wird der Gasfluss abhängig vom Durchmesser der kleinen Atemwege und ist damit unabhängig von Anstrengung und Kraft des Probanden oder Patienten. Diese Abhängigkeit beginnt bei $25 \%$ des ausgeatmeten Volumens (Bates et al. 1971). Alle Parameter, die zu einem späteren Zeitpunkt der maximalen Flussvolumenkurve erfasst werden, beziehen sich auf den aktuellen Widerstand der kleinen Atemwege. Die (Abbildung 7) zeigt das Flussvolumen-Diagramm bei einem Patienten mit einer chronischobstruktiven Lungenerkrankung (COLD).

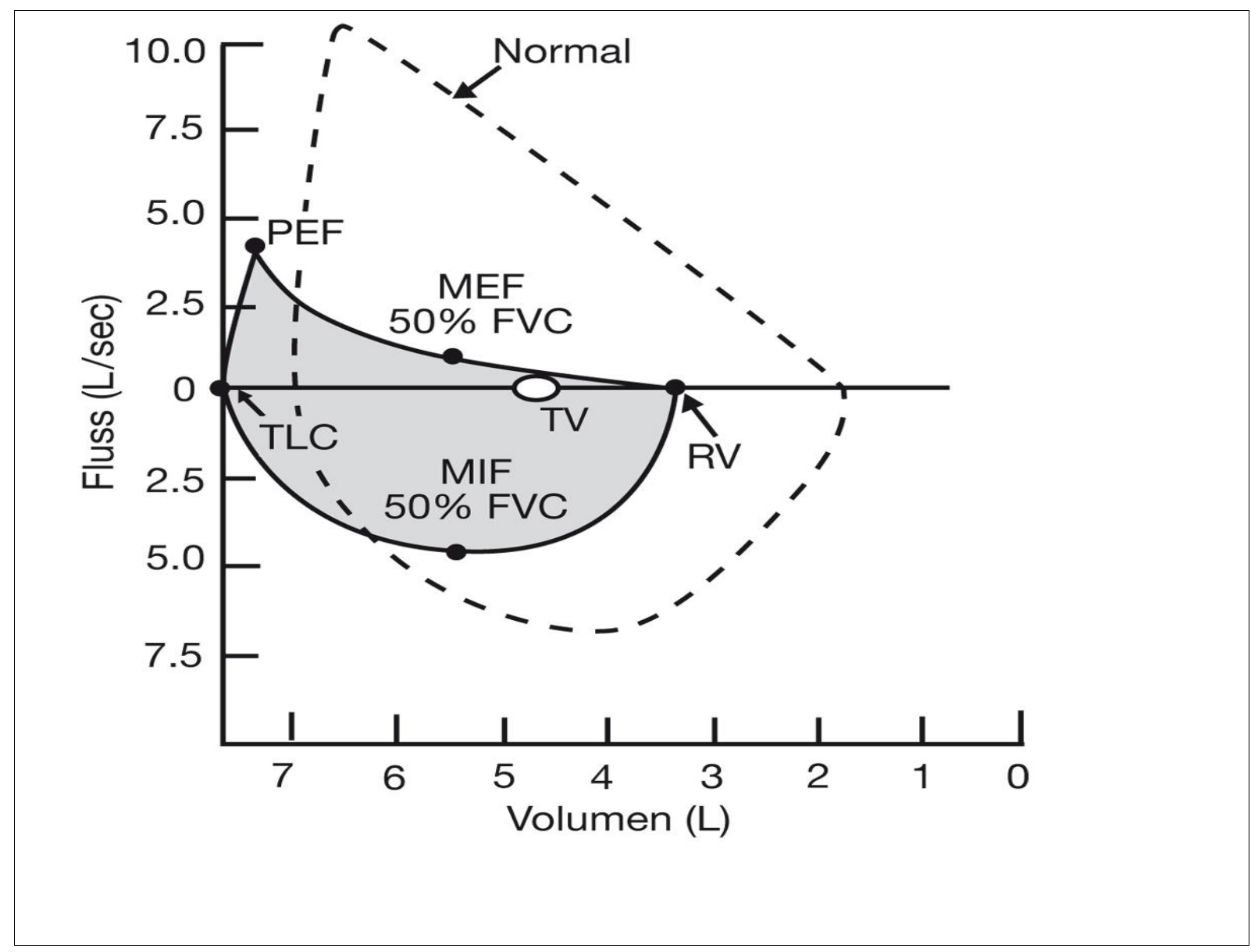

Abbildung 7:

Flussvolumendiagramm einer Person mit fortgeschrittener chronischer Bronchitis. Der maximale exspiratorische Gasfluss nach 50\% der Ausatmung (MEF $50 \%$ FVC) ist wesentlich stärker eingeschränkt als beim Gesunden (Abb. 6) und auch als die Gasbewegung der Inspiration. Die als Fläche erkennbare Atemreserve ist deutlich kleiner als in Abb.6, besonders für die Exspiration. Der MEF $50 \%$ ist ein guter motivationsunabhängiger Parameter zur Kontrolle der obstruktiven Ventilationsstörung. 
Der maximale Gasfluss nach 50\% des ausgeatmeten Volumens (MEF50) ist ein sehr empfindlicher Parameter für das Ausmaß der Obstruktion der peripheren Atemwege. Dies trifft für den PEF (peak expiratoy flow), den maximalen Gasfluss der gesamten forcierten Exspirationskurve nicht zu. Er wird zu einem sehr frühen Zeitpunkt der forcierten Exspiration gemessen und ist damit kraft- und motivationsabhängig.

Seit Wert liegt darin, als Screening-Parameter die aktuelle Form der Flussvolumenkurve ungefähr zu erfassen und dies mit der klinischen Symptomatik in Beziehung setzen zu lassen. Die Flussvolumenkurve der maximalen Inspiration hat eine mehr symmetrische Form als die der maximalen Exspiration. Dies bedeutet, dass in der Inspiration im Gegensatz zur Exspiration der maximale Gasfluss nach der Hälfte des eingeatmeten Volumens erfolgt (MIF50) und nicht früher wie der Spitzenfluss bei der Exspiration. PEF und MIF50 haben keine spezifische Aussagekraft für eine chronisch-obstruktive Erkrankung.

\subsubsection{Die Ganzkörperplethysmographie}

Eine weitere Methode zur Messung der Lungenfunktion ist die Ganzkörper- oder Bodyplethysmographie. Mit dieser Methode kann der Atemwegswiderstand quantitativ erfasst werden. Das Problem einer Widerstandsmessung ist es, den Luftdruck in den Lungenbläschen zu bestimmen, der die gemessene Luftströmung durch die Bronchien auslöst. Der bronchiale Atemwegswiderstand ergibt sich aus dem Quotienten Alveolardruck $\left(\Delta \mathrm{P}_{\mathrm{A}}\right)$ durch die Atemstromstärke $\left(\mathrm{V}^{\prime}\right)$ :

\section{$\Delta P_{A}$ \\ V}

Während die Atemstromstärke (V') spirometrisch bestimmt werden kann, ist die Messung des Alveolardrucks $\left(\Delta \mathrm{P}_{\mathrm{A}}\right)$ erheblich schwieriger.

Erst durch die Ganzkörperplethysmographie ist eine kontinuierliche Registrierung des Alveolardrucks möglich geworden. 


\subsubsection{Das Messprinzip der Ganzkörperplethysmographie}

Der Proband befindet sich während der Messung mit dem ganzen Körper (daher Ganzkörper- bzw. Bodyplethysmographie) in einer knapp $1 \mathrm{~m}^{3}$ großen Kammer, die luftdicht verschließbar ist. Er atmet angewärmte und angefeuchtete Luft aus einem Atembeutel, oder einem elektronischen System ein und aus, das den Atembeutel simuliert. Die Atemluft wird zur Ermittlung der Atemstromstärke (V') in- und exspiratorisch durch einen Flusssensor geleitet und somit wie bei der Spirometrie bestimmt. Die Druckänderungen in der Kammer werden durch einen Drucksensor in der Kammer erfasst und geben im Prinzip ein Negativ der Alveolardruckänderung wieder. (Abbildung 8)

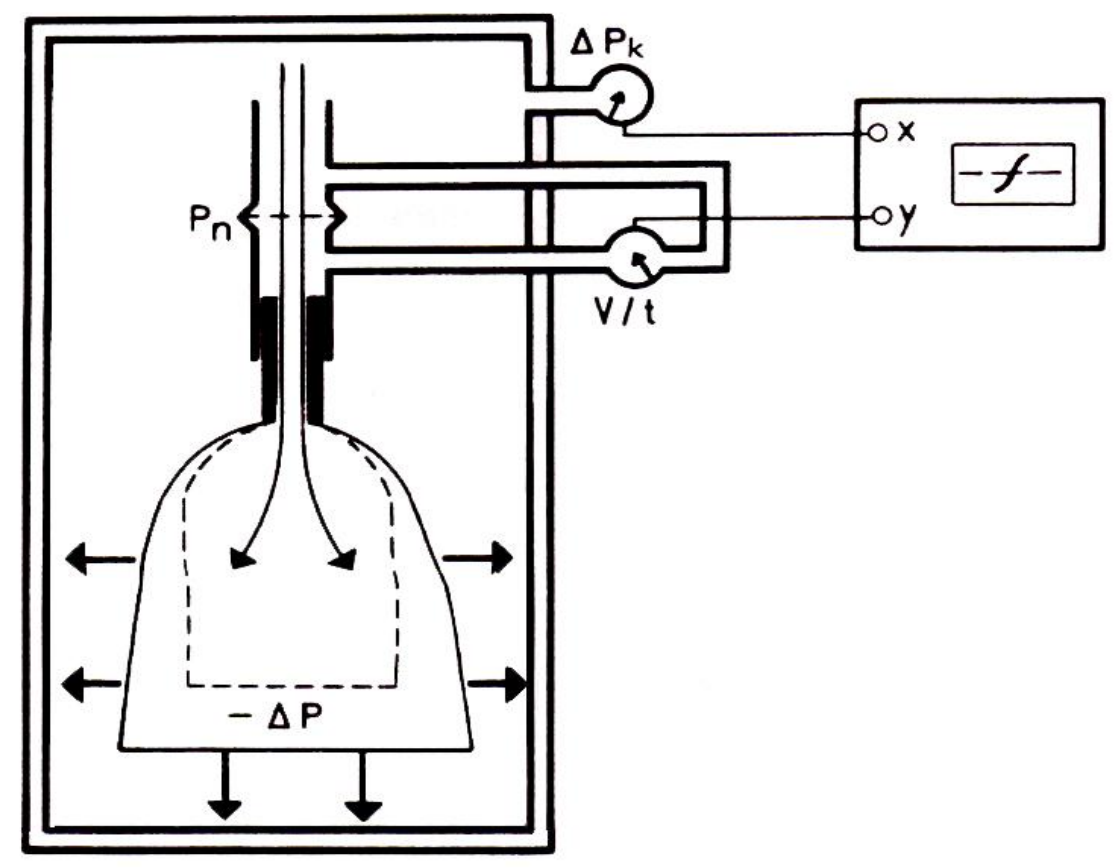

Abbildung 8:

Vereinfachte schematische Darstellung der Bodyplethysmographie mit einem Probanden, der in der Kammer über ein offenes Atemrohr atmet. Gemessen wird der Kammerdruck $\Delta P_{K}$ sowie über den Pneumotachographen $P_{N}$ die Atemstromstärke. Die Atembewegungen des Brustkorbs erzeugen respirationsabhängige Kammerdruckveränderungen. 
Damit die Luft aus der Kammer in die Lungen strömen kann, muss durch die Atemmuskulatur ein negativer Druck in den Alveolen $(-\Delta \mathrm{P})$ erzeugt werden. Dabei wird die Luft in den Alveolen "gedehnt“ und die Lungen nehmen einen etwas größeren Raum in der Kammer ein. Im Gegenzug wird die Kammerluft leicht komprimiert und der Kammerdruck steigt um den Betrag $\Delta \mathrm{P}_{\mathrm{K}}$ an. Dieser Wert liegt bei einem gesunden Menschen in der Größenordnung von ungefähr 1/13,3 kPa $(\sim 1 / 100 \mathrm{mmHg})$. Eine Messung dieses Drucks ist daher nur mit sehr empfindlichen Drucksensoren möglich. Die gemessenen Werte werden in einem 2Koordinatensystem gegeneinander aufgetragen. Die Atemstromstärke wird auf der yAchse und der Kammerdruck auf der x-Achse dargestellt. Das so entstehende Druck-Strömungs-Diagramm erlaubt noch keine Aussage über den bronchialen Strömungswiderstand, da es statt des Alveolardrucks den Kammerdruck enthält. Um die Kurve richtig auswerten zu können, muss bekannt sein, welcher Alveolardruck $\left(\Delta \mathrm{P}_{\mathrm{A}}\right)$ einer bestimmten Kammerdruckänderung $\left(\Delta \mathrm{P}_{\mathrm{K}}\right)$ entspricht. Es ist also erforderlich, die Beziehung $\Delta \mathrm{P}_{\mathrm{A}} / \Delta \mathrm{P}_{\mathrm{K}} \mathrm{zu}$ ermitteln. Dafür ist es notwendig, Messbedingungen zu schaffen, die es ermöglichen, Kammerdruck und Alveolardruck geichzeitig gegeneinander zu registrieren. (Abbildung 9)

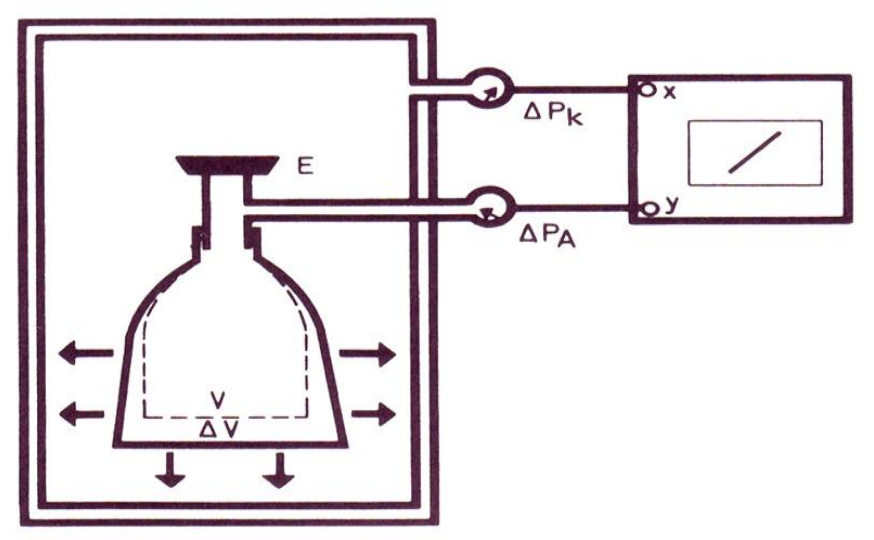

Abbildung 9:

Schematische Darstellung der Messung der Verschlussdruckkurve bei geschlossenem Atemrohr. Bei atemähnlichen Bewegungen des Brustkorbs fällt der Munddruck etwas ab und der Kammerdruck steigt an. Registriert man die Größen „Alveolardruck“ und „Kammerdruck“ gegeneinander, so erhält man die Verschlussdruckkurve. Die Resistance wird mithilfe der Verschlussdruckkurve aus der DruckStrömungs-Kurve berechnet. Mit diesen Daten erfolgt unter Einsatz der Eichkonstante die Berechnung des intrathorakalen Gasvolumens. 
Der Alveolardruck kann direkt am Mundstück gemessen werden, wenn das Atemrohr durch ein Ventil verschlossen wird. In diesem Fall besteht keine Strömung und somit auch kein Druckgefälle mehr zwischen Alveolen und Mundöffnung. An allen Stellen zwischen Alveolen und Mundstück herrscht unter diesen „statischen Bedingungen“ der gleiche Druck. Macht der Proband jetzt atemähnliche Bewegungen, fällt der Alveolardruck, der dem Munddruck entspricht, etwas ab, während der Kammerdruck etwas zunimmt. Registriert man die unter diesen Bedingungen gemessenen Größen $\Delta \mathrm{P}_{\mathrm{A}}$ und $\Delta \mathrm{P}_{\mathrm{K}}$ wiederum auf einem 2-Koordinatensystem direkt gegeneinander, so erhält man eine Gerade, die Verschlussdruck- oder „Shutter-Kurve“. Deren Steigung (tan $\alpha$ ) gibt das Verhältnis $\Delta \mathrm{P}_{\mathrm{A}} / \Delta \mathrm{P}_{\mathrm{K}}$ wieder.

Die Resistance lässt sich nun mit Hilfe der Verschlussdruckkruve aus der DruckStrömungs-Kurve berechnen.

Die Steigung der Druck-Strömungskurve lässt sich beschreiben als: $V / \Delta P_{K}=\tan \beta$.

Die Steigung der Verschlussdruckkurve lässt sich beschreiben als: $\Delta P_{A} / \Delta P_{K}=\tan \alpha$.

Der Quotient aus den Steigungen beider Kurven ergibt die Resistance:

$$
R(\text { Einheit: }[\mathrm{kPa} / \mathrm{lxs}])=\begin{array}{cc}
\tan \alpha \\
-\cdots \\
\tan \beta
\end{array} \quad \begin{gathered}
\Delta P_{A} \\
-\cdots
\end{gathered}
$$




\subsubsection{Intrathorakales Gasvolumen und funktionelle Residualkapazität}

Das Residualvolumen (RV Einheit [l]) entspricht jenem Volumen, das nach maximaler Exspiration noch in der Lunge verbleibt (Abbildung 4). Dieses Lungenvolumen ist nicht ausatembar und kann nur mittels Fremdgasmethoden oder Ganzkörperplethysmographie bestimmt werden.

Bei den Fremdgasmethoden wird ein Fremdgas, z.B. Helium, in Mischung mit Luft eingeatmet. Nach einer gewissen Zeit kommt es zu einer Verteilung des Heliums auf den ventilierten Alveolarraum. Aus der Anfangs- und Endkonzentration des Heliums und des Spirometertotraumes lässt sich die funktionelle Residualkapazität nach einem einfachen Dreisatz berechnen. Die Messergebnisse beziehen sich grundsätzlich auf den ventilierten Alveolarraum. Intrathorakale Gasmengen, die nicht an der Ventilation teilnehmen, z.B. in Emphysemregionen oder im Pneumothorax, können mit den Fremdgasmethoden grundsätzlich nicht erfasst werden, wohl aber mit der Bodyplethysmographie.

Wird das Atemrohr in der Kammer genau am Ende einer normalen Ruheexspiration geschlossen, so entspricht das intrathorakale Volumen (IGV) genau der funktionellen Residualkapazität (FRC). Die plethysmographische Bestimmung des IGV beruht auf dem Prinzip des Boyle-Mariotte-Gesetzes. Während der Atembewegungen ist das Produkt aus $\mathrm{P} \times \mathrm{V}=$ const..

$\mathrm{V}$ ist das im Moment des Verschlusses im Thorax befindliche Volumen, also das gesuchte intrathorakale Gasvolumen (IGV). Da die Volumen- und Druckänderungen $\Delta \mathrm{V}$ und $\Delta \mathrm{P}$ während der Atembewegungen gegenüber $\mathrm{V}$ und $\mathrm{P}$ sehr klein sind, kann das Differential der Boyle-Mariotte-Gleichung gebildet werden:

$d P \times V+d V \times P=0$

$$
V=\frac{d V}{d P}
$$

oder für endliche Zustandsänderungen:

$$
I G V=-\frac{\Delta V}{\Delta P_{A}}
$$


Die registrierte Shutter-Kurve gibt die Beziehung zwischen $\Delta \mathrm{P}_{\mathrm{K}}$ und $\Delta \mathrm{P}_{\mathrm{A}}$ wieder. Von $\Delta \mathrm{P}_{\mathrm{K}}$ kann man leicht auf die gesuchte Größe $\Delta \mathrm{V}$ schließen, indem man durch Eichung feststellt, um welchen Betrag der Kammerdruck ansteigt, wenn z.B. durch Eichung ein definiertes Luftvolumen in die Kammer gefüllt wird.

$\Delta V=\Delta P_{K} \times$ Eichkonstante

Dann gilt für das intrathorakale Gasvolumen:

$$
\begin{array}{cl}
\Delta P_{K} & \text { Eichkonstante } x[(\text { atm. Druck }) \\
\Delta P_{A} & (k P a)-(6,27 \mathrm{kPa})] .
\end{array}
$$

\subsection{Gemessene Lungenfunktionsparameter}

\section{1-Sekundenkapazität $\left(\mathrm{FEV}_{1}\right)$}

Die Einsekundenkapazität $\mathrm{FEV}_{1}$ (engl. Forced Expiratory Volume in 1 Second) ist die nach maximaler Einatmung in einer Sekunde rasch ausgeatmete Luftmenge (siehe Abb. 4 und 5). Es handelt sich um einen sehr wichtigen dynamischen Lungenfunktionsparameter, der traditionell in (I) angegeben wird, obwohl er eigentlich die Dimension der Geschwindigkeit hat. Die Bezugsgröße für die Einsekundenkapazität sind die in größeren Populationen gewonnenen Normalwerte für diesen Parameter.

\section{Die forcierte Vitalkapazität (FVC)}

Die FVC (I und \% des Normalwertes) entspricht dem vollständig ausgeatmeten Lungenvolumen beim gleichen Atemmanöver, d.h. rasche Ausatmung nach tiefer Einatmung. Sie wird in der Regel so bestimmt, kann aber auch bei langsamer Einoder Ausatmung erfasst werden, wenn dafür eine Indikation besteht. Krankheitsprozesse wie eine chronische Obstruktion schränken die FVC ein. (Abbildung 5). Eine restriktive Ventilationsstörung führt auch zur FVC-Abnahme, allerdings nicht durch Einschränkung der Atemdynamik wie in Abbildung 5, sondern mit Abnahme aller in Abbildung 4 dargestellten Lungenvolumina durch den Krankheitsprozess. 


\section{FEV $_{1} /$ FVC}

Das Verhältnis von FEV 1 zu FVC kann als Beziehung der aktuellen Messgrößen (Tiffenau-Wert, FEV 1 als \%FVC) bzw. zur Frage, ob dieses Verhältnis den in größeren Populationen erhobenen Normalwert erreicht, dargestellt werden. Wenn er erreicht wird, beträgt er 100\% (\% predicted). Der Tiffenau-Wert ist ein sehr wichtiger praktisch nutzbarer Parameter für Lungengesundheit bzw. für das Ausmaß einer obstruktiven Ventilationsstörung. Ein Vergleich der Strömungsverhältnisse in Abbildung 4 und 5 bei gesunden und chronisch-obstruktiven Strömungsverhältnissen zeigt, dass der Parameter bei beginnender Obstruktion, wenn die FVC noch nicht so stark eingeschränkt ist, als Maß für zunehmende Abweichung von der Norm bzw. Gesundung genutzt werden kann. Dieser diagnostische Wert verliert sich bei zunehmender Obstruktion, da der Blähungszustand auch deutlich zunimmt, was die Unterschiede zwischen FEV 1 und FVC kleiner werden lässt.

\section{Maximale exspiratorische Strömungsgeschwindigkeit (PEF)}

Die maximale exspiratorische Strömungsgeschwindigkeit (Peak Expiratory Flow, I/s) entspricht dem während der Exspiration erreichbaren maximalen Gasfluss. Dieser Parameter ist im Flussvolumen-Diagramm erkennbar (Abbildung 6 und 7). Er kann mittels einfacher Geräte als Screening-Parameter zur respiratorischen Befindlichkeit von lungenkranken Patienten selbst ermittelt werden.

\section{Die funktionelle Residualkapazität (FRC)}

Die FRC (I und \% predicted) kann beim kooperationsfähigen Patienten sehr schnell mit dem Bodyplethysmographen erfasst werden (Abbildung 8 und 9). Die Gasmenge enspricht dem Volumen, das während der Exspirationsstellung der normalen Ruheatmung im Thorax vorhanden ist. Die Messmethode erfasst im Gegensatz zu den Fremdgasmethoden auch Gasmengen, die im Thorax vorhanden sind, nicht jedoch an der Ventilation teilnehmen. Die FRC hat eine Bedeutung für alle Prozesse der respiratorischen Pathophysiologie. 


\section{Residualvolumen (RV)}

Das Residualvolumen entspricht der Gasmenge, die nach tiefer Exspiration noch in der Lunge verbleibt (I und \% predicted). Die vorliegenden Messwerte wurden mit der Bodyplethysmographie erfasst, wobei nach Bestimmung der FRC das exspiratoriche Reservevolumen subtrahiert wurde.

\section{Das totale Lungenvolumen (TLC)}

Das totale Lungenvolumen entspricht der Summe von VC und RV (I und \% predicted). Es kann daher nur mit Methoden erfasst werden, mit denen auch das RV erfasst werden kann.

\section{Die Resistance (R)}

Die Resistance entspricht dem bronchialen Strömungswiderstand $\left(\mathrm{cm} \mathrm{H}_{2} \mathrm{O} \times \mathrm{I}^{-1} \times \mathrm{s}\right)$. Beim Lungengesunden hat die registrierte Kurve eine Form, die einer geraden Linie sehr nahe kommt. Ist der Wert erhöht, beobachtet man häufig eine Schleifenform, bei der z.B. In- und Exspiration getrennt bewertet werden können. Die Resistance ist ein sehr empfindlicher Parameter für eine freie oder durch einen Krankheitsprozess gestörte Gasströmung. 


\subsection{Die elektrische Impedanztomographie}

Bei der elektrischen Impedanztomographie (EIT) handelt sich es um eine bildgebende Methode, die transversale Querschnittsbilder des menschlichen Körpers nichtinvasiv und ohne Strahlenbelastung erzeugen kann. Die EIT ermöglicht es, die Verteilung der elektrischen Impedanz im Inneren von nicht homogenleitfähigen Körpern zu ermitteln und aus den gewonnenen Daten Bilder zu generieren. Das Prinzip der EIT beruht auf der Messung von elektrischen Eigenschaften biologischer Gewebe. Die theoretische Voraussetzung zur Erstellung von EIT-Bildern ist durch die bekannte gewebsspezifische Heterogenität der elektrischen Bioimpedanz unterschiedlicher Gewebe und Organe gegeben.

\begin{tabular}{|c|c|}
\hline Gewebe & Impedanz ( $\Omega \times \mathrm{cm})$ \\
\hline Blut & 150 \\
\hline Lunge & $700-2400$ \\
\hline Fett & $2000-2700$ \\
\hline Knochen & 16600 \\
\hline Muskel & 125 längs \\
\hline Muskel & 1800 quer \\
\hline Herzmuskel & 160-575 längs \\
\hline Herzmuskel & $420-5200$ quer \\
\hline
\end{tabular}

Tabelle 2:

Elektrische Impedanzen biologischer Gewebe (nach Geddes und Baker 1967, S. 280)

Erstmalig beschrieben wurde das Messprinzip der EIT Mitte der achtziger Jahre von Brown und Barber (Brown und Barber 1989). Das Verfahren basiert auf der Messung von Spannungen auf der Oberfläche der untersuchten Körperregion nach Einspeisung eines bekannten niedrigen Wechselstroms (Harris et al. 1987). Die Stromeinspeisung und Messung der resultierenden Oberflächespannungen erfolgt rotierend über Oberflächenelektroden, die zirkulär in einer Ebene auf der zu untersuchenden Körperregion angebracht sind. Aus den gemessenen Spannungswerten und dem eingespeisten Messstrom wird die Verteilung des elektrischen Widerstands im Inneren des untersuchten Objekts bzw. Körperteils berechnet und als Bild dargestellt. 
Die Erzeugung eines tomographischen Impedanzverteilungsbildes aus den gewonnenen Daten wird Bildrekonstruktion genannt.

Bei Anwendung der EIT im thorakalen Bereich erfolgen die Applikation des Wechselstroms und die nachfolgende Messung der entstehenden Oberflächenspannung über 16 Hautelektroden, die in einer Ebene symmetrisch und zirkulär um den Brustkorb verteilt sind. Die oberflächlich messbaren Spannungen sind dabei abhängig von der Verteilung des elektrischen Widerstands im Brustkorb, der Bioimpedanz.

Während der Messung wird über jeweils ein benachbartes Elektrodenpaar der insgesamt 16 Elektroden der Wechselstrom appliziert, wobei an den verbleibenden 13 Elektrodenpaaren, die nicht an der Stromeinspeisung beteiligt sind, die resultierenden Oberflächenspannungen gemessen werden. Nach der ersten Stromeinspeisung und den dazugehörigen 13 Messungen wird das benachbarte Elektrodenpaar zur Stromeinspeisung genutzt. Ein einzelnes EIT-Bild kann erstellt werden, wenn ein rotierender Zyklus aus insgesamt 16 Stromeinspeisungen und den dazugehörigen 13 Messungen der Oberflächenspannung pro Stromeinspeisung abgeschlossen ist. Das bedeutet, dass in einem kompletten Messzyklus, der den Brustkorb umläuft, insgesamt 208 (13 x 16) einzelne Oberflächenspannungen gemessen werden. Die Datenerfassung kann mit einer Frequenz von bis zu 45 Messzyklen pro Sekunde erfolgen, was im Prinzip 45 Einzelbildern entspricht.

Das Messprinzip der EIT ist schematisch in Abbildung 10 dargestellt.

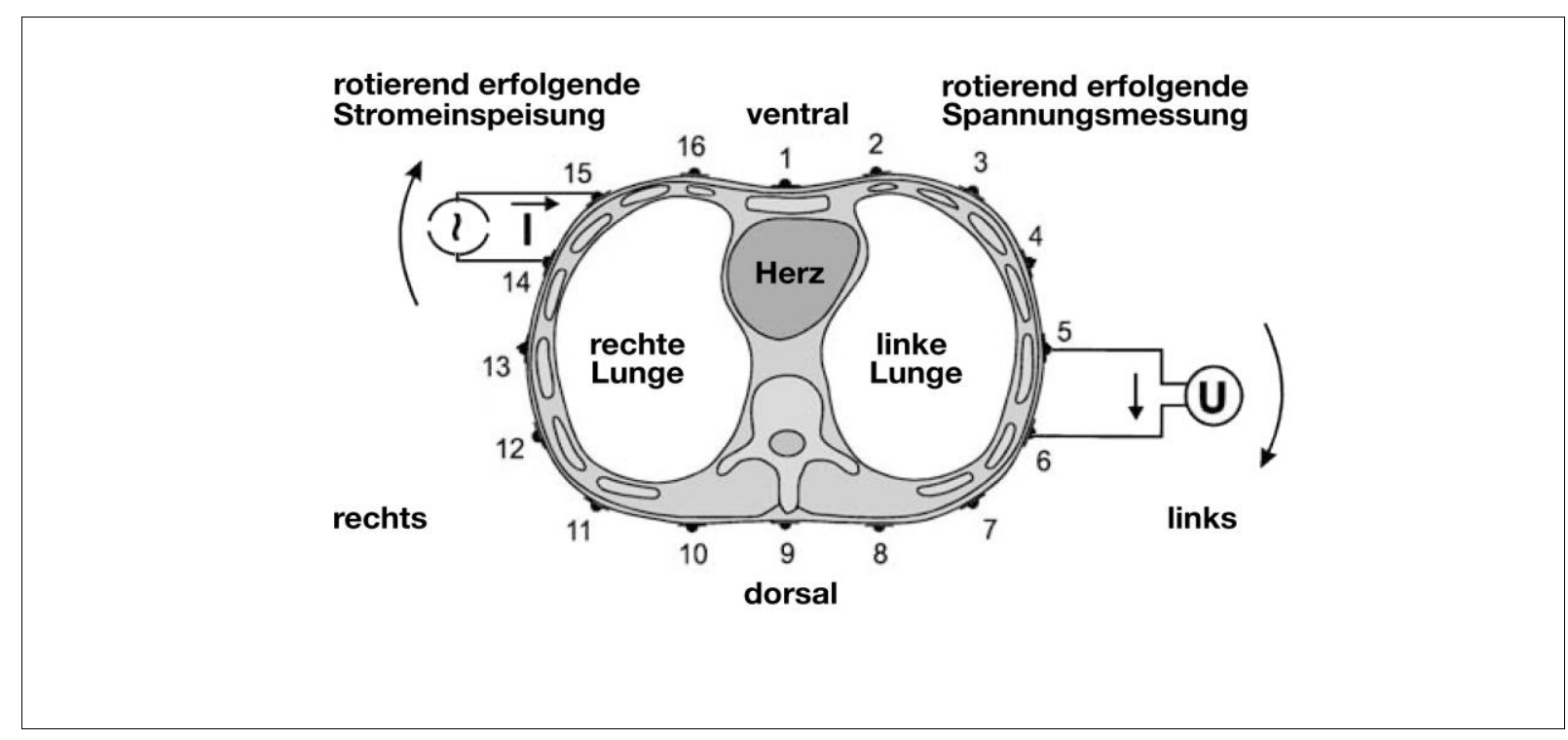

Abbildung 10:

Schematische Darstellung des Messprinzips der EIT bei einer EIT-Untersuchung am Thorax. 
Das eigentliche EIT-Bild wird durch einen Rückprojektionsalgorithmus (Brown und Barber 1989) aus den 208 gemessenen Oberflächenspannungen in der Bildrekonstruktion erzeugt. Dazu wird zunächst jede der 208 gemessenen Oberflächenspannungen auf die mittlere Oberflächenspannung während der Messung normalisiert. Aus diesen normalisierten Oberflächenspannungen wird mit dem Algorithmus ein Tomogramm errechnet, das ein Auflösungsvermögen von $32 \mathrm{x}$ 32 Pixel hat.

Somit entsteht aus jedem einzelnen Messzyklus ein zweidimensionales Bild, das die Verteilung der elektrischen Impedanz im Thoraxquerschnitt zeigt.

Im Gegensatz zu anderen bildgebenden Verfahren wie der konventionellen radiologischen Aufnahme, der Computertomographie, oder der Magnetresonanztomographie liefert die EIT keine morphologisch-anatomischen Bilder. Computertomographie und Magnetresonanztomographie erzeugen Schnittbilder mit hoher regionaler Auflösung und liefern somit Informationen von anatomischen Strukturen bzw. morphologischen Gegebenheiten im untersuchten Objekt. Die EIT erzeugt zweidimensionale Bilder, die Aufschluss über die Verteilung und Änderung des elektrischen Widerstands im untersuchten Objekt liefern.

Es konnte gezeigt werden, dass für den Großteil des elektrischen Widerstands im Thorax die in den Lungen enthaltene Luft verantwortlich ist (Hahn et al. 1996, Frerichs et al. 2002).

Eine geringere Bedeutung im Hinblick auf den intrathorakalen Widerstand nehmen pulsatiler Blutfluss und intrathorakale Flüssigkeitsverschiebungen ein.

Somit besteht ein direkter Zusammenhang zwischen intrathorakalem Luftgehalt und elektrischem Widerstand im Thorax. $\mathrm{Da}$ sowohl physiologische als auch pathologische Veränderungen des Luft- und Flüssigkeitsgehalts zu großen Veränderungen der regionalen elektrischen Impedanz führen, ist die EIT in der Lage, die regionale Gasverteilung, sowie mögliche örtliche Schäden des Lungenparenchyms zu erfassen. Ein wesentliches Merkmal der EIT ist das hohe zeitliche Auflösungsvermögen der Methode. Wie oben erwähnt, ist es möglich bis zu 45 Einzelbilder pro Sekunde zu erstellen und das über einen beliebig langen Zeitraum. Die etablierten bildgebenden Verfahren zur Erstellung von Querschnittsbildern erzeugen statische Bilder, die zwar eine hohe Auflösung bieten, aber lediglich den Zeitpunkt der Untersuchung widerspiegeln, was im Prinzip einem Schnappschuss entspricht. 
EIT-Untersuchungen, die über längere Zeitintervalle durchgeführt werden, ermöglichen es, Änderungen der elektrischen Gewebeeigenschaften zu erfassen, die durch eine Veränderung der Struktur oder Funktion des untersuchten Organs hervorgerufen werden. Aufgrund des hohen Zeitauflösungsvermögens ist es aber ebenso möglich, Veränderungen zu erfassen, die sich in relativ kurzen Zeitfenstern abspielen.

Alle Daten dieser Studie wurden mit dem Göttinger EIT-Gerät Goe-MF-II (Goe-MF-II System, Anästhesiologische Forschung, Universität Göttingen, Deutschland (Hahn et al. 2002)) erhoben. Es handelt sich hierbei um ein Messsystem, das erstmals im Jahr 2003 eingesetzt wurde. Die Frequenz des eingespeisten Wechselstroms kann vom Benutzer aus einem Bereich zwischen $5-500 \mathrm{kHz}$ gewählt werden.

Der für diese Untersuchungen eingespeiste Strom hatte eine Stromstärke von $5 \mathrm{~mA}(\mathrm{rms})$ und eine Frequenz von $50 \mathrm{kHz}$. Die gewählte Bildfrequenz betrug $25 \mathrm{~Hz}$. Es wurden in dieser Studie somit 25 komplette Messzyklen pro Sekunde aufgezeichnet, wobei insgesamt 1000 Messzyklen pro Messung durchgeführt wurden. Eine Messung dauerte somit genau 40 Sekunden.

\subsubsection{Protokoll der Messungen}

Zur Messung wurden den Probanden 16 EKG-Elektroden (BR-50-K, Medicotest, Ølstykke, Dänemark) zirkulär in Höhe der Brustwarzen um den Thorax geklebt. Um eine symmetrische Verteilung der Elektroden zu erreichen, wurden zunächst vier Elektroden geklebt, die den Thoraxumfang im Prinzip gevierteilt haben. Jeweils eine der vier Elektroden wurde dementsprechend ventral auf dem Sternum, dorsal auf der Wirbelsäule, sowie beidseitig in der mittleren Axillarlinie aufgeklebt. Die nächsten vier Elektroden wurden in die Mitte der entstandenen Zwischenräume geklebt. Die verbliebenen acht Elektroden wurden mit derselben Methode genau in die Mitte der acht neuen Zwischenräume geklebt.

Bei einigen Probanden mussten die Stellen für die Elektroden vorher rasiert werden, um einem optimalen Kontakt zwischen Elektrode und Haut zu gewährleisten. Nach dem Aufkleben der Elektroden wurden diese mit dem EIT-Gerät verbunden. Die ventrale Elektrode über dem Sternum ist die erste Elektrode. Die anderen Elektroden (2-16) wurden der Reihe nach gegen den Uhrzeigersinn mit dem EIT-Gerät verbunden (Abbildung 10). 
Die Probanden wurden in vier verschiedenen Körperlagen untersucht. Als erstes wurden die Probanden im Sitzen, danach im Liegen, sowie in rechter und linker Seitenlage untersucht. Die Reihenfolge der Seitenlagen wurde zufällig gewählt. In jeder dieser Körperlagen mussten die Probanden vier verschiedene Atemmanöver durchführen. Das erste gemessene Atemmanöver war eine normale Spontanatmung in Ruhe. Danach folgten zwei Vitalkapazitätsmanöver. Zunächst wurden die Probanden gebeten, ein forciertes Vitalkapazitätsmanöver (FVC) durchzuführen. Dabei sollten die Probanden nach maximaler Einatmung in möglichst kurzer Zeit maximal ausatmen. Beim zweiten Vitalkapazitätsmanöver (VC) wurde nach maximaler Einatmung über einen längeren Zeitraum ausgeatmet. Die maximale Ausatmung von der totalen Lungenkapazität (TLC) bis zum Residualvolumen (RV) dauerte hier zwischen 10-15 Sekunden. Das als letztes durchgeführte Manöver setzte sich aus drei verschiedenen Spontanatmungsphasen zusammen. Begonnen wurde mit normaler Spontanatmung. Danach mussten die Probanden maximal, also bis zum Residualvolumen ausatmen. Spontanatmung in dieser Phase bedeutet ein normales Atemzugvolumen einzuatmen und bei der nächsten Ausatmung erneut bis zum Residualvolumen auszuatmen. Die letzte Phase dieses Manövers startete mit maximaler Einatmung bis zur totalen Lungenkapazität. Von der TLK ausgehend mussten die Probanden ein normales Atemzugvolumen ausatmen, um mit der nächsten Inspiration erneut die TLC zu erreichen. Wie oben erwähnt, wurden insgesamt 1000 Bilder mit einer Frequenz von $25 \mathrm{~Hz}$ aufgezeichnet. Die Aufzeichnung der einzelnen Atemmanöver konnte somit über einen Zeitraum von genau 40 Sekunden erfolgen. Im Fall des letzten Manövers standen für die normale Spontanatmung, die Spontanatmung nahe dem RV, sowie für die Spontanatmung nahe der TLC dementsprechend zirka 10 Sekunden pro Phase zur Verfügung. Alle Atemmanöver wurden vor den Messungen eingehend mit den Probanden besprochen und geübt. Die Probanden konnten die Ihnen bekannten Manöver während der Messungen somit auf Kommando ausführen. 


\subsubsection{Auswertung der EIT-Daten}

Die Verarbeitung der gewonnenen EIT-Daten zu Bildern, die ausgewertet werden können, erfolgt nach den Messungen. Im ersten Schritt dieser "off-line“ Datenverarbeitung müssen die relevanten Phasen der Messungen extrahiert werden. Diese Phasen beinhalten die ungestörte Spontanatmung, die maximale Exspiration von der TLC zum RV während der Vitalkapazitätsmanöver (FVC, VC), sowie die Spontanatmung auf drei unterschiedlichen Lungenvolumenniveaus.

Somit wird nicht die Gesamtzahl von 1000 Bildern bzw. Scans pro Messung in die Weiterverarbeitung einbezogen, sondern nur die Anzahl an Bildern, die das jeweilige Atemmanöver widerspiegeln.

Die selektierten Daten wurden genutzt, um sogenannte funktionelle EIT-Bilder der regionalen Lungenventilation zu erstellen und um eine quantitative Aussage über die Gasverteilung zwischen rechter und linker Lunge treffen zu können.

\subsubsection{Erstellung von funktionellen EIT-Bildern}

Mit der funktionellen EIT lassen sich Brustkorbquerschnittsbilder erstellen, die die regionale Ventilation, bzw. die Änderung des regionalen Lungenvolumens über einen Zeitraum abbilden. Um funktionelle EIT-Bilder erstellen zu können, ist die Aufzeichnung von EIT-Daten über längere Zeitintervalle notwendig. Das Verfahren beruht auf einer Kompression von Serien einzelner EIT-Bilder bzw. der gemessenen regionalen Impedanzänderung. Aus einem langen EIT-Datensatz, der mehrere hundert, oder auch tausende einzelner EIT-Bilder enthalten kann, wird durch die Anwendung eines relativ einfachen Auswertungsalgorithmus ein einziges funktionelles EIT-Bild erstellt. Die Erzeugung eines funktionellen EIT-Bildes ist schematisch in Abbildung 11 dargestellt. 


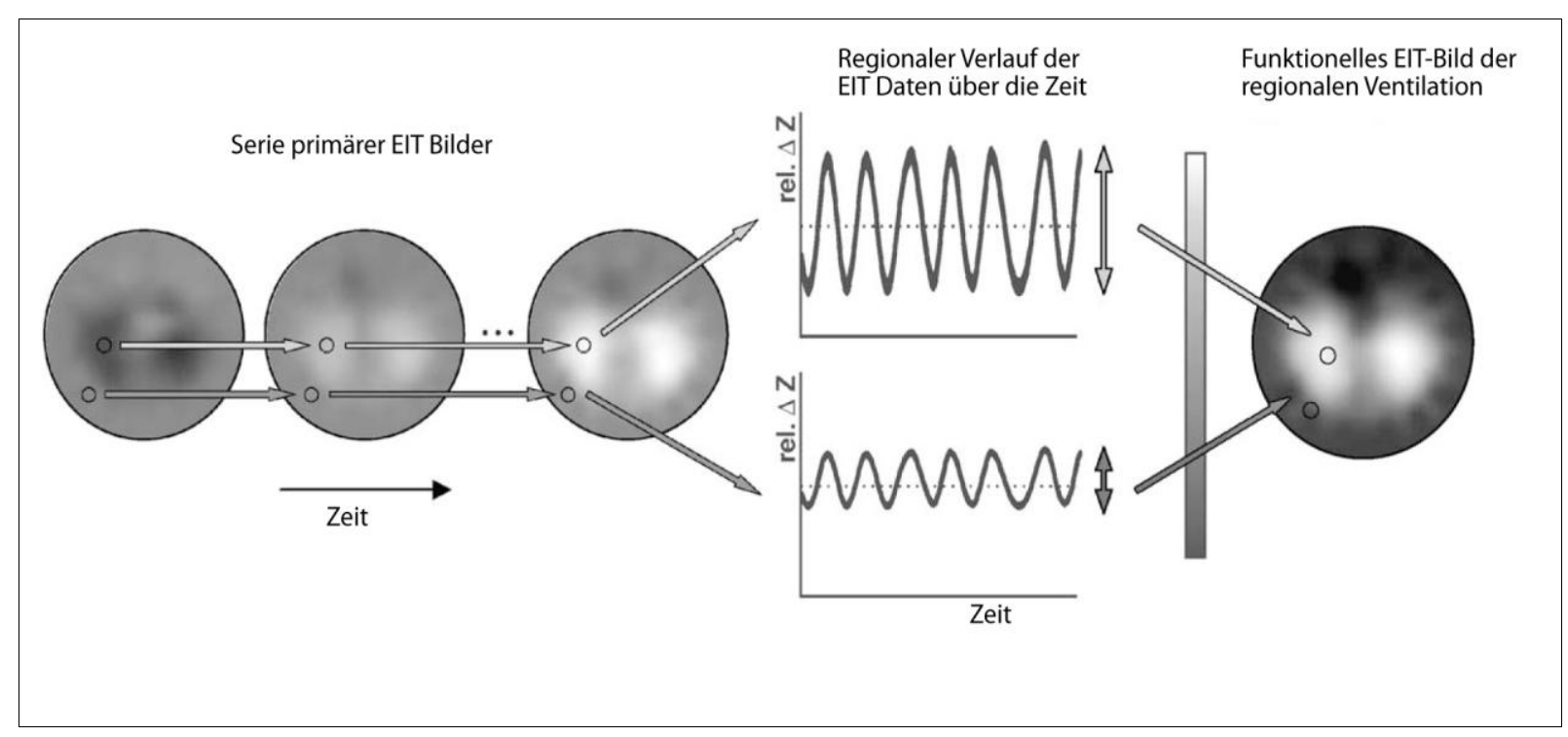

Abbildung 11:

Erzeugung eines funktionellen EIT-Bildes.

Ziel dieser Datenauswertung ist es, eine Aussage über die regionale Gasverteilung in der Lunge treffen zu können, die dem dynamischen Prozess der Atmung Rechnung trägt. Das regionale Lungenvolumen variiert in Abhängigkeit vom Atemzyklus stark mit der Zeit. Die aufgezeichneten Zeitverläufe der regionalen Impedanzänderung geben die atmungsbedingten Änderungen der regionalen Luftfüllung in der Lunge wieder und sind somit ein Maß für die regionale Ventilation. Der oben genannte Auswertungsalgorithmus errechnet die Standardabweichung der regionalen Impedanzänderung für jeden der 912 Bildpunkte aus der EIT-Bildserie. Regionen mit hoher Ventilation weisen eine hohe Standardabweichung auf, solche mit weniger Ventilation zeigen geringere Variationen. Durch Bestimmung der regionalen Differenz zwischen den maximalen und minimalen Werten, der relativen Impedanzänderung, die periodisch am Ende jeder Ein- bzw. Ausatmung erreicht werden, ist es z.B. möglich, die regionale Gasbewegung bzw. die regionale Ventilation zu bestimmen. 


\subsection{Statistik}

Die im Text und in den Graphiken angegebenen Werte sind Mittelwerte \pm Standardabweichung. Die Signifikanz der Unterschiede zwischen den berechneten Parametern jeder Altersgruppe wurde mit dem Wilcoxon matched pairs signed rank test ermittelt. In den Diagrammen sind die signifikanten Unterschiede zwischen der sitzenden und der liegenden Position, sowie die Unterschiede zwischen der liegenden Position und der rechten bzw. der linken Seitenlage dargestellt. Die Signifikanz der Unterschiede zwischen den jungen und alten Probanden wurde mit dem Wilcoxon-Mann-Whitney-u-Test bestimmt. $P<0,05$ wurde als signifikant gewertet. Im Fall von multiplen Vergleichen wurde die Schwelle der Signifikanz mit der Bonferroni-Methode festgelegt. 


\section{Ergebnisse}

\subsection{Konventionelle Lungenfunktion}

Die Tabelle 1 zeigt die Werte der Lungenfunktionsuntersuchung aller Probanden einschließlich der Mittelwerte. Es handelt sich ganz überwiegend um normale Funktionswerte, die als Referenzwerte für eine ungestörte bronchiale Durchlässigkeit und normale Volumenverhältnisse angesehen werden können. Bei je einem Probanden in beiden Gruppen finden sich Zeichen einer veränderten Resistancekurve in Richtung auf eine diskrete Obstruktion (j04 und a03). Der ältere Proband wies mit FEV1/FVC 0,65 eine beginnende Obstruktion auf. Da aber alle übrigen Funktionsdaten keinerlei Einschränkungen aufweisen, sind die bronchialen Reserven hier nur leicht eingeschränkt und zwar für beide Gruppen gleich, so dass ein Einschluss dieser Probanden in die Untersuchungen gerechtfertigt ist. Die altersbedingte Einschränkung der aktuellen Lungenfunktionsdaten im Vergleich der jungen gegen die ältere Gruppe, etwa bei FEV1 und FVC, ist nur zum Teil auf die Lungenalterung zurückzuführen. Die verbleibenden Differenzen sind dadurch bedingt, dass beide Gruppen unterschiedliche Körperproportionen aufweisen und ein unterschiedliches Niveau der Referenzwerte erreichen, in jungen Jahren meist über $100 \%$, im Alter oft darunter. Ein sicheres Zeichen der Lungenalterung ist das als Ausmaß des Blähungszustandes bezeichnete Verhältnis von RV zu TLC, das bei den jungen Probanden durchschnittlich 20,9\% aufweist, bei den älteren $43,9 \%$.

\subsection{EIT-Untersuchungen}

\subsubsection{Spontanatmung in Ruhe}

Während der normalen Spontanatmung in Ruhe wies die regionale Ventilation in der rechten und linken Lunge unabhängig von der Körperposition und der Altersgruppe nur sehr geringe Unterschiede auf. Lediglich die örtliche Belüftung der linken Lunge war bei den jungen Probanden in der rechten Seitenlage deutlich geringer. Die entsprechenden EIT-Daten sind graphisch in Abbildung 12 dargestellt. 


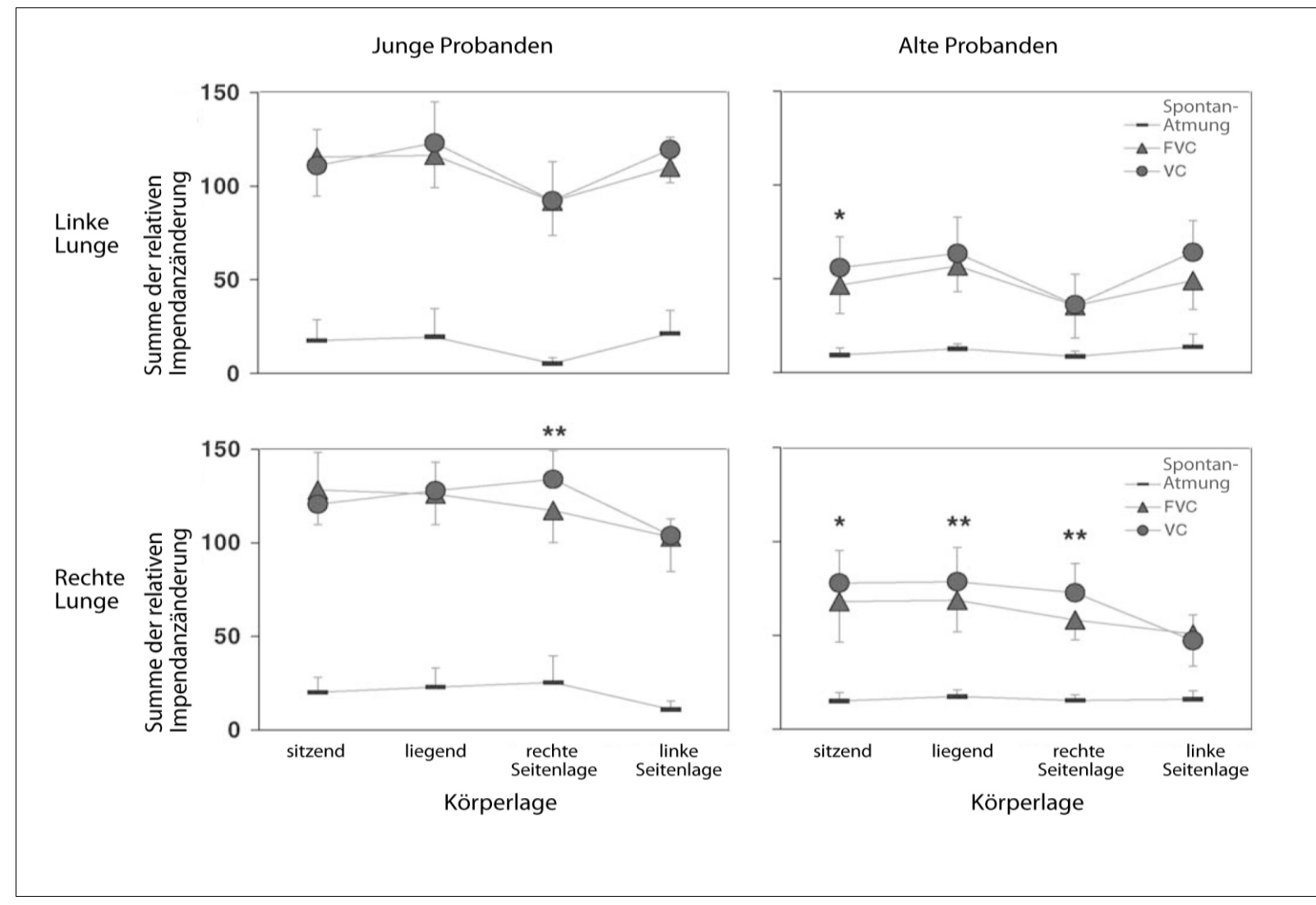

Abbildung 12:

Regionale Änderungen des Gasvolumens in der linken und rechten Lungenregion bei einer Gruppe junger und alter Probanden, während normaler Spontanatmung, forcierter Exspiration (FVC) und langsamer Exspiration und vier verschiedenen Körperpositionen. Dargestellt ist die Summe der relativen Impedanzänderung von Endinspiration zu Endexspiration. Aufgetragen sind Mittelwerte \pm Standardabweichung. Die Sterne markieren signifikante Unterschiede zwischen FVC und VC. ${ }^{*} \mathrm{P}<0,05,{ }^{* *} \mathrm{P}<0,01$.

In diesen vier kartesischen Koordinatensystemen ist die jeweilige Körperlage der Probanden auf der Abszisse dargestellt. Auf der Ordinate ist die Summe der relativen Impedanzänderung von Endinspiration zu Endexspiration während der Atemmanöver aufgetragen. Hohe Änderungen der relativen Impedanz sind mit hoher Ventilation gleichzusetzen, niedrigere Werte lassen auf eine geringere Belüftung schließen. Die beiden links angeordneten Koordinatensysteme beziehen sich auf die jungen, die rechts angeordneten enthalten die Werte der alten Probanden. Eine gesonderte Betrachtung der linken und rechten Lunge ist durch eine weitere Unterteilung der Ergebnisse möglich. Die beiden oberen Diagramme zeigen nur die gemessenen Werte der linken Lunge, die Ergebnisse der rechten Lunge sind in den beiden unteren Grafiken ersichtlich. 
In jedem dieser vier Koordinatensysteme sind drei Kurven enthalten, die drei der vier verschiedenen Atemmanöver darstellen.

Es steht jeweils eine Kurve für die Spontanatmung in Ruhe (markiert mit Rechtecken), eine für die forcierte Exspiration FVC (markiert mit Dreiecken) und eine für die langsamen Exspirationsmanöver VC (markiert mit Kreisen).

Der relative Anteil, den die rechte Lunge an der Gesamtventilation einnimmt, hat sich in der vermessenen Ebene bei Lagewechsel von sitzender zu liegender Position weder bei den jungen noch bei den alten Probanden signifikant verändert. Die graphische Darstellung dieser Aussage findet sich in Abbildung 13.

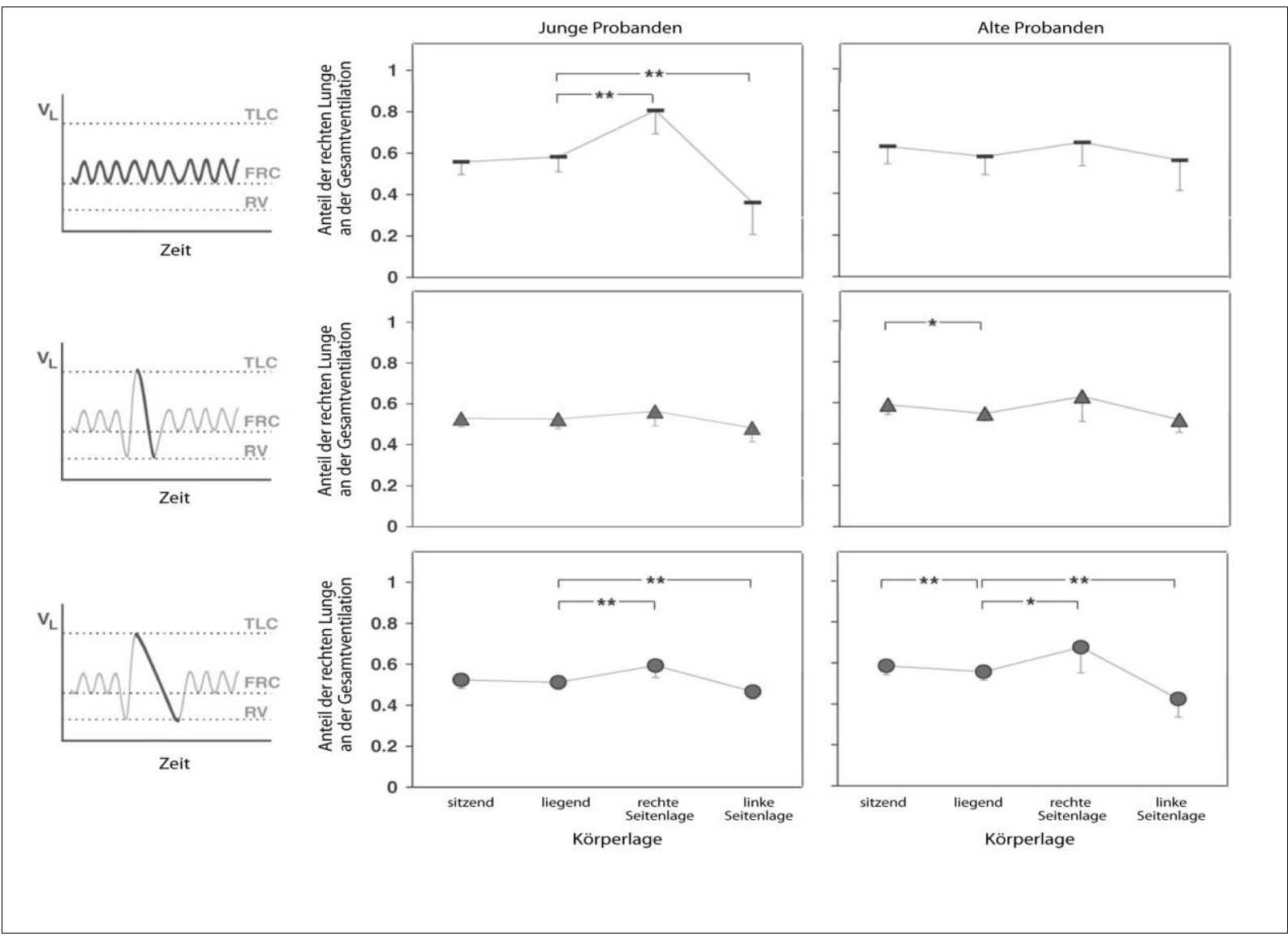

Abbildung 13:

Dargestellt ist der Anteil, den die rechte Lunge an der Gesamtventilation bei den jungen und alten Probanden in der vermessenen Ebene während normaler Spontanatmung (oben), forcierter Exspiration (Mitte) und langsamer Exspiration (unten) in vier verschiedenen Körperpositionen einnimmt. Aufgetragen sind Mittelwerte \pm Standardabweichung. Die kleineren Diagramme, die links angeordnet sind bilden schematisch die Atmenmanöver ab. Die in die Analyse einbezogenen Phasen der Messungen sind durch dicke Striche hervorgehoben. $\mathrm{V}_{\mathrm{L}}=$ Lungenvolumen, $\mathrm{TLC}=$ Totale Lungenkapazität, FRC = Funktionelle Residualkapazität, $R V=$ Residualvolumen. ${ }^{*} \mathrm{P}<0,05,{ }^{*} \mathrm{P}<$ 0,01 . 
In diesen sechs Koordinatensystemen ist die jeweilige Körperlage der Probanden wie zuvor auf der Abszisse aufgetragen. Auf der Ordinate kann der Anteil, den die rechte Lunge an der Gesamtventilation hat, abgelesen werden. Die Einteilung von 0 bis 1 entspricht einem Anteil von 0 bis $100 \%$. Die Daten der jungen Probanden finden sich wie zuvor in den linken Graphiken. Die Daten der alten Probanden sind in den rechten Graphiken enthalten. Wie in Abbildung 12 sind auch in Abbildung 13 nur die drei ersten der insgesamt vier verschiedenen Atemmanöver dargestellt. Eine Zuordnung der unterschiedlichen Untersuchungsserien zu den Atemmanövern ist durch eine Markierung wie in Abbildung 13 möglich. Zur besseren Orientierung findet sich links neben den Koordinatensystemen zusätzlich eine grafische Darstellung der Atemmanöver. Die Änderung des Lungenvolumens ist hier schematisch gegen die Zeit aufgetragen.

Ebenfalls deutlich zu erkennen ist, dass bei den jungen Probanden die rechte und die linke Seitenlage jeweils zu einer deutlichen Zunahme der Ventilation in der unten liegenden Lunge führt. In der Gruppe der älteren Probanden zeigte sich während der normalen Spontanatmung in der vermessenen Ebene in keiner der Positionen eine signifikante Lageabhängigkeit der Ventilation. Dieses unterschiedliche Verhalten der regionalen Ventilation bei jungen und alten Probanden in Abhängigkeit der Erdanziehungskraft ist in Abbildung 14 als funktionelles EIT-Bild der regionalen Ventilation für einen jungen und einen alten Probanden dargestellt. 


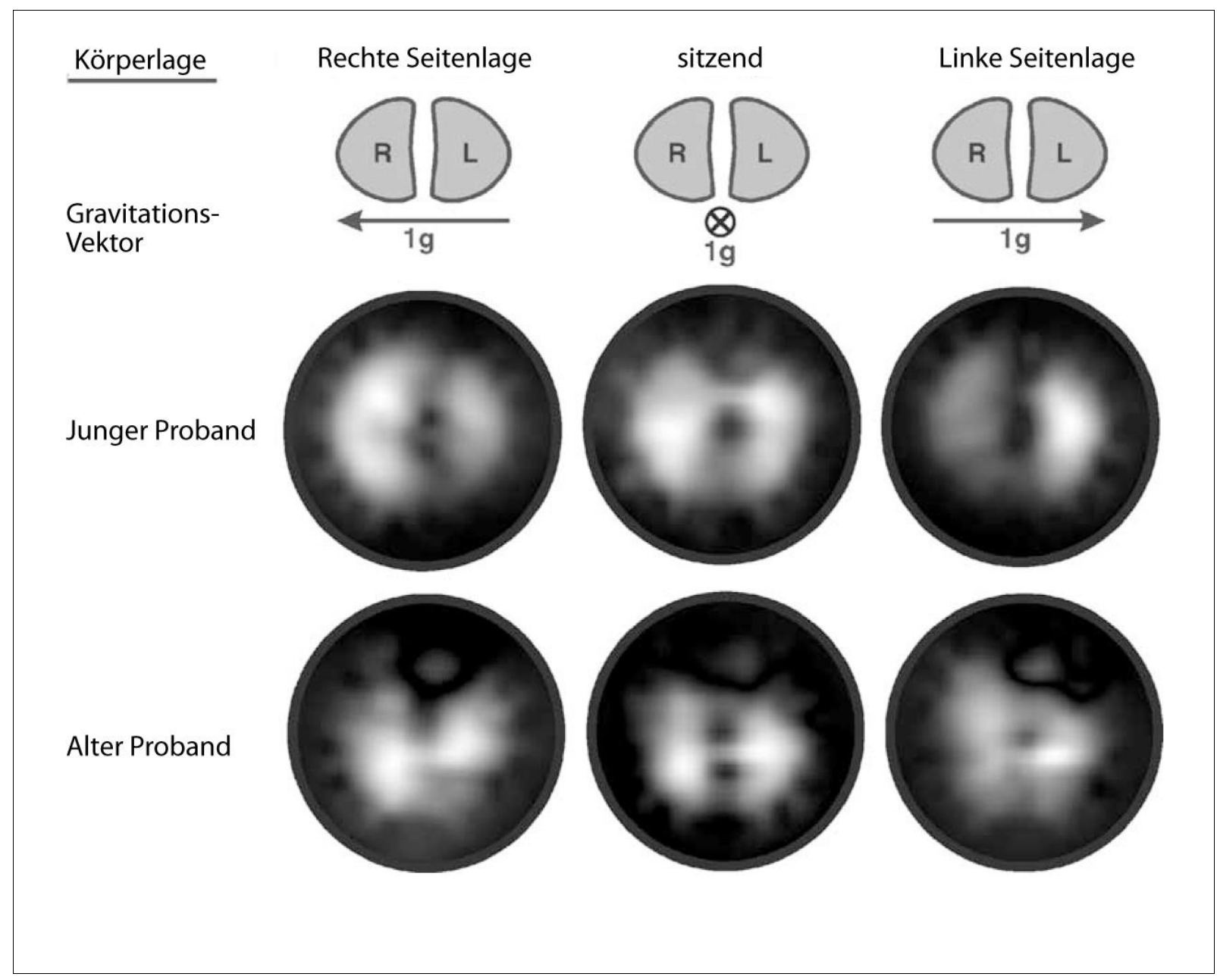

Abbildung 14:

Funktionelle elektrische Impedanztomographie der regionalen Lungenventilation eines 25 Jahre alten (oben) und eines 73 Jahre alten gesunden Probanden (unten) während normaler Spontanatmung im Sitzen, sowie in rechter und linker Seitenlage. Je mehr Ventilation stattfindet je heller stellt sich das entsprechende Areal im funktionelle EIT-Bild dar. Die Bilder sind als sagittale Schnittbilder zu verstehen. Der obere Teil des Bildes zeigt die ventrale Region, der linke Teil des Bildes zeigt die rechte Körperseite (Frerichs et al. 2001a).

In der sitzenden Position wirkt die Erdanziehungskraft senkrecht auf die vermessene Ebene, was zu einer symmetrischen Gasverteilung in der rechten und linken Lunge bei den jungen sowie bei den alten Probanden führt.

In beiden Seitenlagen wirkt die Erdanziehungskraft parallel zu der vermessenen Ebene. Bei den jungen Probanden führte dies zu einer deutlich höheren Ventilation der unten liegenden Lunge sowohl in rechter, als auch in linker Seitenlage. Diese von der Gravitation abhängige Gasverteilung in der Gruppe der jungen Probanden konnte in der Gruppe der alten Probanden nicht beobachtet werden. 


\subsubsection{FVC und VC}

In beiden Altersgruppen waren während der Vitalkapazitätsmanöver FVC und VC die relativen Impedanzänderungen (Abbildung 12) deutlich größer als bei der Spontanatmung in Ruhe. Bei beiden Manövern FVC und VC sind in allen Körperlagen die gemessenen Werte der jungen Probanden deutlich höher als die Werte der alten Probanden. Innerhalb der Altersklassen führt ein Vergleich der beiden Atemmanöver FVC und VC zu folgendem Ergebnis: Insgesamt sind die gemessenen Werte der beiden Manöver im Fall der jungen Probanden fast identisch. Der einzige signifikante Unterschied zwischen den Werten beider Manöver zeigt sich in der rechten Lunge, in der rechten Seitenlage. Hier lässt sich erkennen, dass die gemessenen Werte des VC-Manövers deutlich über den Werten des FVC-Manövers liegen. In der linken Lunge kann man in linker Seitenlage ein ähnliches Verhalten erkennen, das sich aber weniger signifikant darstellt. In der Gruppe der alten Probanden kann man ein ähnliches Verhalten in beiden Seitenlagen erkennen. Im Fall der alten Probanden sind die Unterschiede zwischen beiden Atemmanövern allerdings in beiden Seitenlagen hoch signifikant. Die gemessenen Werte des VCManövers liegen in der jeweils unten liegenden Lunge deutlich über den Werten des FVC- Manövers. Darüber hinaus zeigen sich bei den alten Probanden auch in sitzender und in liegender Position deutlich höhere VC- als FVC-Werte. Für die jeweils oben liegende Lunge sind die gemessenen Werte in den Seitenlagen in beiden Altersklassen, während beider Manöver fast identisch.

Der Anteil, den die rechte Lunge in der vermessenen Ebene an der Gesamtventilation einnimmt, ist für beide Manöver ebenfalls in Abbildung 13 dargestellt. Während des FVC-Manövers stellte sich bei den jungen Probanden keine lageabhängige Gasverteilung zwischen der rechten und linken Lunge dar. Bei den alten Probanden sank der Anteil der rechten Lunge an der Gesamtventilation bei einem Lagewechsel in die liegende Position. Im Fall des VC-Manövers war der Anteil der rechten Lunge an der Gesamtventilation bei den alten Probanden im Liegen ebenfalls reduziert. Verglichen mit der liegenden Position hat die jeweils unten liegende Lunge in den Seitenlagen den größten Anteil an der Gesamtventilation.

Ein Vergleich des lokalen endexspiratorischen Volumens nach den Vitalkapazitätsmanövern FVC und VC mit dem Volumen der funktionellen Residualkapazität ist in Abbildung 15 grafisch ersichtlich. 


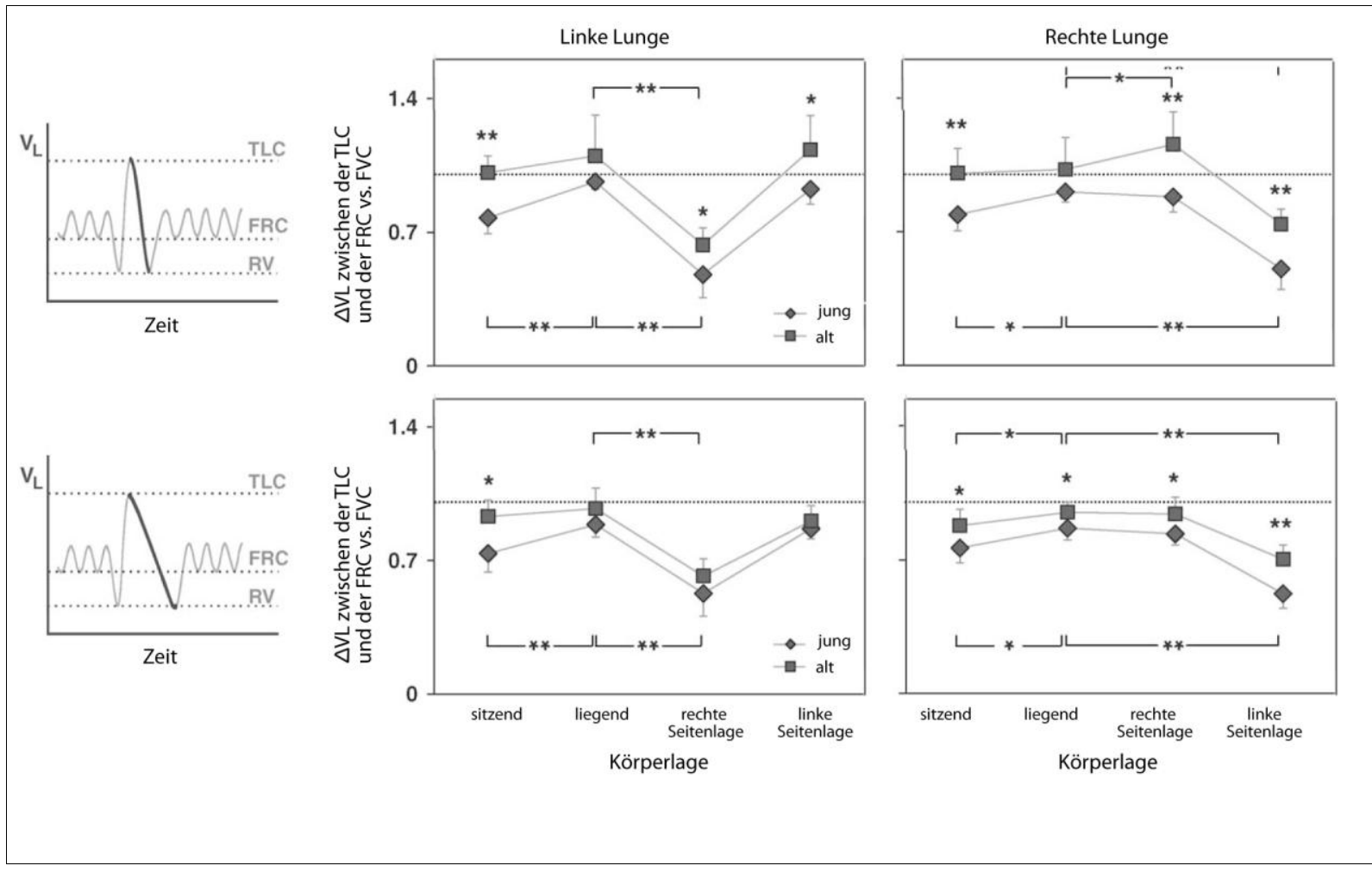

Abbildung 15:

Vergleich der Lungenvolumenveränderung, die zwischen der TLC und der FRC auftritt, mit der Lungenvolumenänderung, die während der FVC- und VC-Manöver auftritt. Dargestellt sind die Werte der zugehörigen Summen der relativen Impedanzänderung von Endinspiration zu Endexspiration. Aufgetragen sind Mittelwerte \pm Standardabweichung. Die Daten stammen aus den Atemmanövern FVC (oben) und VC (unten) der jungen und alten Probanden in den vier verschiedenen Körperlagen. Die zusätzlich in die Diagramme eingezeichnete fein gepunktete Linie markiert den Zahlenwert 1. Dieser Wert zeigt an, ob die Volumenänderung zwischen TLC und FRC mit der Volumenänderung während FVC und VC identisch ist. Zahlenwerte über 1 zeigen an, dass die Entleerung der Lunge oberhalb der normalen FRC stoppt. Diese Verhalten zeigt sich bei den alten Probanden während der FVC-Manöver. Die Sterne oberhalb der Kurven zeigen signifikante Unterschiede zwischen den jungen und alten Probanden. Die Sterne im oberen und unteren Bereich der Diagramme zeigen statistisch signifikante Unterschiede innerhalb der Gruppen von alten und jungen Probanden. * $\mathrm{P}<$ $0,05,{ }^{* *} P<0,01$.

Die Berechnung der Werte basiert auf der Änderung des Lungenvolumens zwischen der TLC und der FRC, sowie der Volumenänderung während der FVC- und VCManöver. Die Körperlage ist in diesen Grafiken wiederum auf der Abszisse aufgetragen. Auf der Ordinate kann im Fall der beiden oberen Graphiken die Differenz des Lungenvolumens zwischen funktioneller Residualkapazität und lokalem endexspiratorischem Volumen nach der forcierten Exspiration bei dem FVC-Manöver abgelesen werden. 
Im Fall der beiden unteren Graphiken kann die Volumendifferenz zwischen FRC und endexspiratorischem Volumen nach dem VC-Manöver abgelesen werden. Zur besseren Orientierung sind die beiden Atemmanöver auch hier links neben den Graphiken schematisch dargestellt. Die beiden linken Graphiken beziehen sich auf die Werte der linken Lungen. Die Werte der rechten Lungen sind in den beiden rechten Koordinatensystemen ersichtlich. Im Gegensatz zu den anderen Darstellungen sind die Werte der jungen und alten Probanden in Abbildung 15 nicht getrennt dargestellt. Die mit einem Quadrat markierten Kurven beziehen sich auf die alten Probanden.

Die mit einem um $45^{\circ}$ gekippten Quadrat markierten Kurven zeigen die Werte der jungen Probanden. Um die Auswertung der Daten zu erleichtern, ist in jedem Koordinatensystem eine fein gepunktete Linie eingezeichnet, die den Zahlenwert 1 auf der Ordinate markiert. Der Wert 1 ergibt sich, wenn die Änderung des Lungenvolumens zwischen der TLC und der FRC der Änderung des Lungenvolumens während der vollständigen Exspirationen bei den Manövern FVC und VC entspricht. Zahlenwerte größer als 1 kommen zustande, wenn nach maximaler Ausatmung ein lokales Volumen in der Lunge verbleibt, das größer ist, als das Volumen der lokalen funktionalen Residualkapazität.

Betrachtet man die Werte detailliert, erkennt man, dass in der Gruppe der alten Probanden die Werte höher sind, als die Werte der jungen Probanden. Allerdings sind die Unterschiede nicht immer signifikant. Darüber hinaus waren bei den alten Probanden die Werte nach dem FVC- Manöver in einigen Körperlagen größer als 1, was bedeutet, dass das regionale endexspiratorische Lungenvolumen nach der maximalen forcierten Exspiration größer war, als das Volumen der regionalen funktionellen Residualkapazität. Bei den jungen Probanden waren die entsprechenden Werte in jedem Fall kleiner als 1. Beide Atemmanöver (FVC und VC) führten bei den jungen Probanden in jeder Körperlage also zu einer Entleerung der Lunge, die unter das Volumenniveau der funktionellen Residualkapazität reicht. Ein deutlicher Anstieg des Wertes stellte sich bei den jungen Probanden bei beiden Manövern beim Lagewechsel von sitzender zu liegender Position ein. Im Fall der alten Probanden kam es hingegen nur zu einem leichten Anstieg des Wertes, der nicht signifikant ist. In beiden Altersgruppen hat der Lagewechsel von liegender Position in eine der Seitenlagen bei beiden Manövern zu einem deutlichen Abfall des Wertes in der jeweils oben liegenden Lunge geführt. In der jeweils unten liegenden Lunge kam es hingegen nicht zu einer Veränderung des Wertes. 
Physiologisch bedeuten diese Erkenntnisse, dass bei beiden Atemmanövern in beiden Seitenlagen die jeweils oben liegende Lunge eine starke Änderungen des regionalen Gasvolumens erfährt und dass das Gasvolumen nach Exspiration unter der funktionellen Residualkapazität liegt. Im Gegensatz dazu endete die Entleerung der jeweils unten liegenden Lunge bei beiden Manövern im Bereich der funktionellen Residualkapazität, bei den jungen Probanden etwas unterhalb der FRC und bei den alten Probanden etwas oberhalb der FRC.

\subsubsection{Spontanatmung auf verschiedenen Lungenvolumenniveaus}

Die typische Charakteristik der EIT-Messung während des letzten Atemmanövers ist in Abbildung 16 dargestellt.

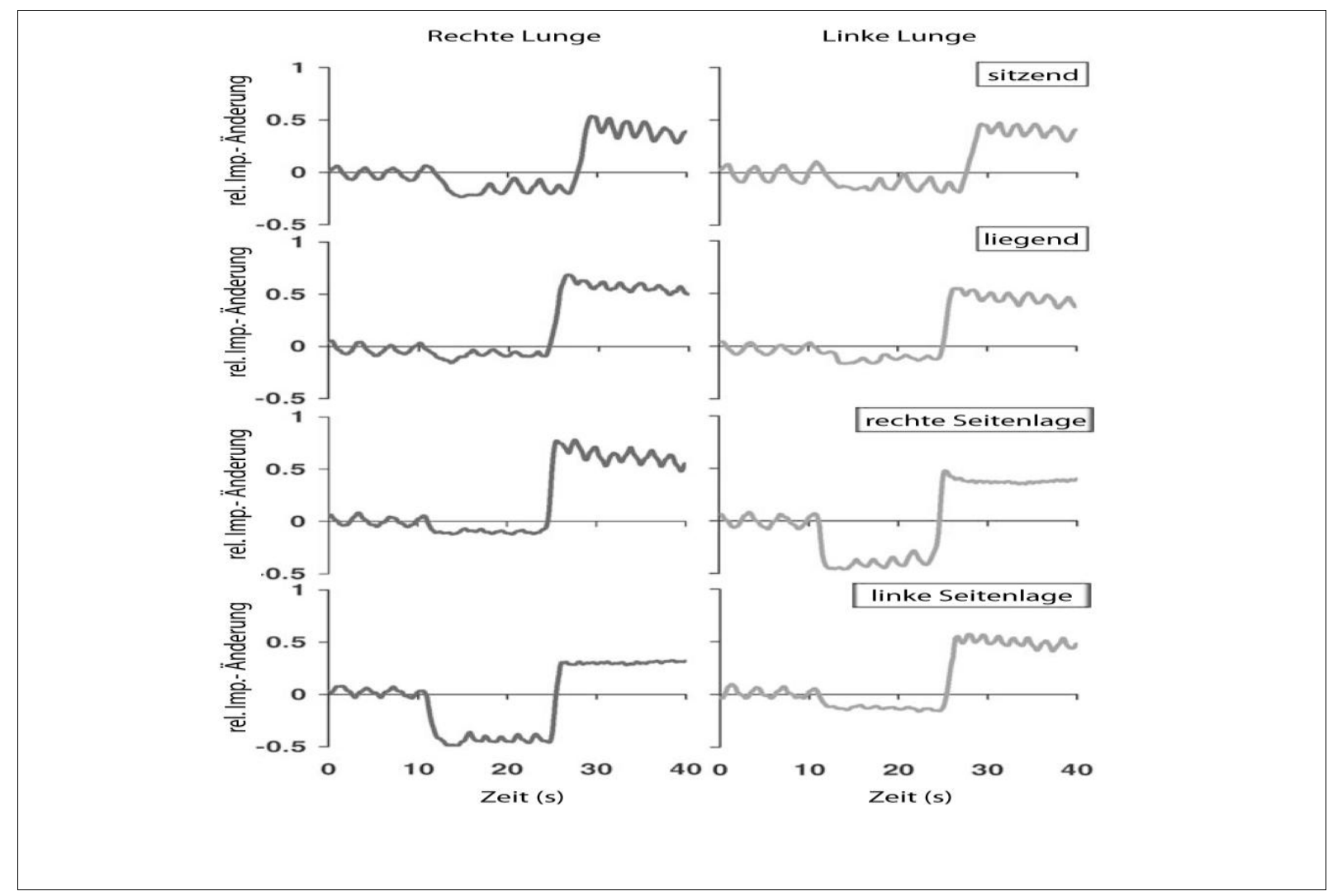

Abbildung 16:

Lokale relative Impedanzänderung in der rechten und linken Lungenregion bei einem spontan atmenden 74 Jahre alten Probanden in vier verschiedenen Körperpositionen. Der Proband atmete zuerst normal spontan, danach mit einem geringen Lungenvolumen (nahe dem RV) und im Anschluss mit einem hohen Lungenvolumen (nahe der TLC). Die Volumenänderung wurden von den Probanden nach einem Drittel und nach zwei Dritteln jeder Messung auf Kommando ausgeführt. Die kleinen Schwankungen der Impedanzkurven geben jeweils die individuellen Atemzüge wieder. 
Die Grafiken stellen die relativen Impedanzänderungen der rechten und linken Lunge eines alten Probanden dar, der auf drei unterschiedlichen Lungenvolumenniveaus spontan atmet. In Abbildung 16 ist auf den Abszissen der Graphiken ein Zeitraum von vierzig Sekunden dargestellt. Dies ist genau der Zeitraum, den jede einzelne Messung gedauert hat. Die Ordinaten zeigen die lokalen Änderungen der regionalen Impedanz. Die links angeordneten Graphiken zeigen die Werte der rechten Lunge. Die Werte der linken Lunge sind in den rechten Graphiken abzulesen. Außerdem sind alle Körperlagen in getrennten Grafiken aufgeführt. Die beiden oberen Grafiken enthalten die Daten der sitzenden, darunter sind die Daten der liegenden Lage abzulesen. Die beiden Seitenlagen sind in den vier unteren Koordinatensystemen dargestellt.

Bei der Auswertung der Daten ist die Abnahme der regionalen Impedanz während der maximalen Ausatmung nach ungefähr einem Drittel der Messung und die Zunahme der regionalen Impedanz bei maximaler Einatmung nach circa zwei Dritteln der Messung deutlich zu erkennen. Ebenfalls deutlich zu erkennen sind die kleineren, zyklischen Änderungen der relativen Impedanz, die der Spontanatmung auf den drei unterschiedlichen Lungenvolumenniveaus entsprechen. In der sitzenden und der liegenden Position ist die relative Änderung der Impedanz für die rechte und linke Lunge sehr ähnlich. Deutliche Unterschiede zeigen sich hingegen bei den Messungen in den beiden Seitenlagen. Der Wechsel von normaler Spontanatmung in Ruhe zu einer Spontanatmung nahe dem Residualvolumen führte nur zu einem geringen Abfall der relativen Impedanz in der unten liegenden Lunge, während sich ein deutlicher Abfall der relativen Impedanz in der oben liegenden Lunge darstellt. Ebenfalls deutlich zu erkennen ist, dass in der unten liegenden Lunge bei Spontanatmung nahe dem Residualvolumen kaum Änderungen der relativen Impedanz stattfinden. Die typischen zyklischen Impedanzänderungen bei Spontanatmung sind hier nur in geringer Ausprägung zu erkennen. In der folgenden Phase der Messung, also der Spontanatmung nahe der totalen Lungenkapazität kommt es zu einem starken Anstieg der relativen Impedanzänderung in beiden Lungen. Verglichen mit dem ersten Drittel der Messung ist dieser Anstieg besonders deutlich in der unten liegenden Lunge zu erkennen. Die quantitativen Ergebnisse dieser Messung sind in Abbildung 17 als Anteil der rechten an der Gesamtventilation der Lunge dargestellt. 


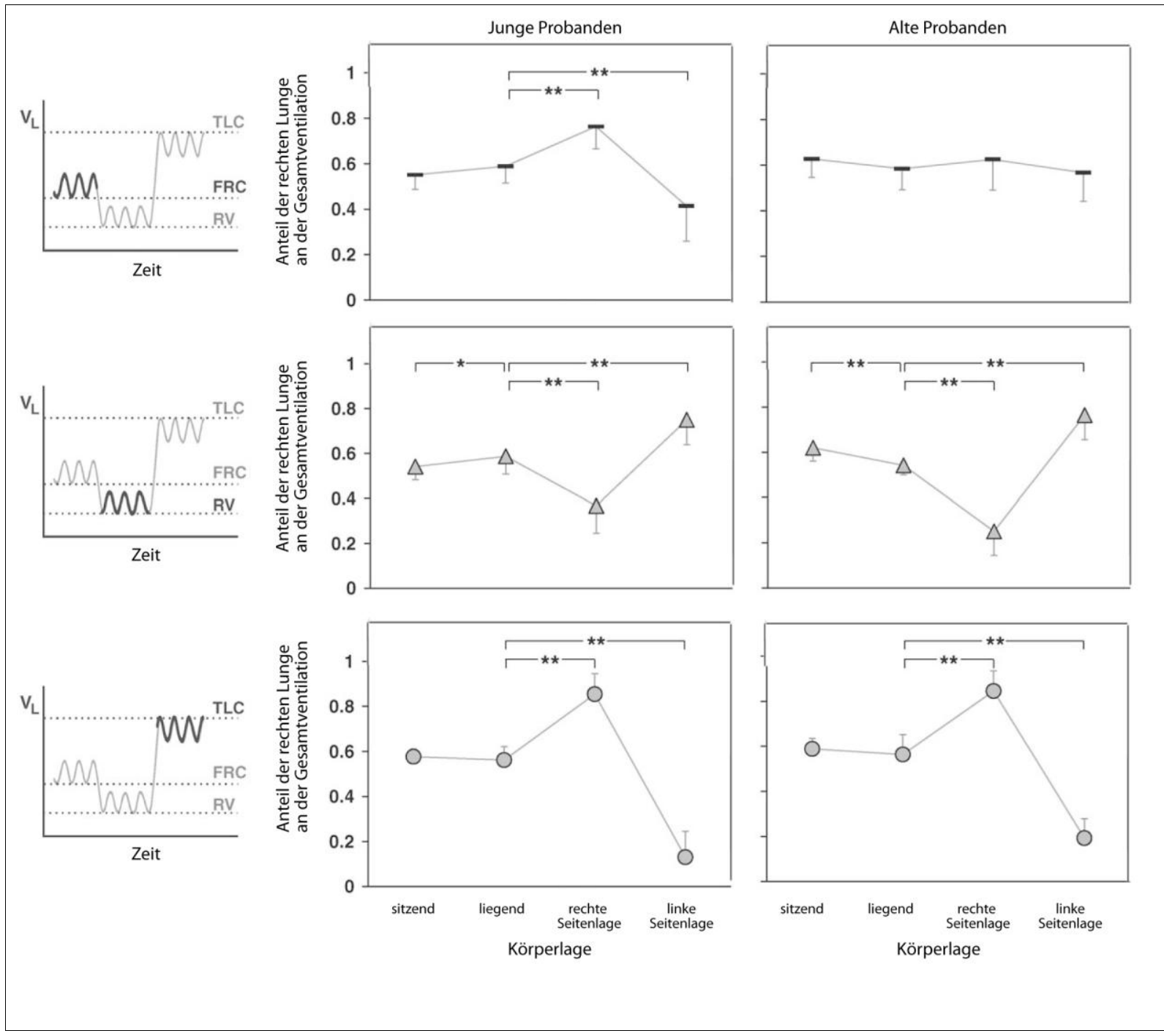

Abbildung 17:

Dargestellt ist der Anteil, den die rechte Lunge an der Gesamtventilation in der vermessenen Ebene bei den jungen und alten Probanden, während normaler Spontanatmung (oben) und während der Atmung mit einem geringen (Mitte) bzw. hohen Lungenvolumen (unten) in vier verschiedenen Körperpositionen einnimmt. Aufgetragen sind Mittelwerte \pm Standardabweichung. Die kleineren Diagramme links veranschaulichen den in die Analyse eingegangenen Teil der Messung. ${ }^{*} P<0,05$, ${ }^{* *} \mathrm{P}<0,01$.

Die Grafiken entsprechen von der Anodnung der Abbildung 13. Die Darstellung ist nach den jeweiligen Atemmanövern sortiert. Die jeweilige Messphase ist schematisch wie zuvor links neben den Graphiken dargestellt.

Die Ergebnisse des ersten Drittels der Messung, also der normalen Spontanatmung entsprechen im wesentlichen der Ergebnisse der Spontanatmung, die in Abbildung 13 dargestellt sind. Somit kann diese erste Phase als eine Art Kontrolle der Ergebnisse der normalen Spontanatmung gesehen werden. 
Die statistische Analyse dieser beiden Messungen ergab keine signifikanten Unterschiede zwischen den unterschiedlichen Messungen der Spontanatmung und unterstreicht somit die gute Reproduzierbarkeit der Ergebnisse. Während der zweiten Phase der Messung (Abbildung 17, mittig) stieg beim Lagewechsel von der sitzenden in die liegende Position bei den jungen Probanden der Anteil, den die rechte Lunge an der Gesamtventilation einnimmt. Im Gegensatz dazu nahm der Anteil, den die rechte Lunge an der Gesamtventilation bei den alten Probanden einnimmt, unter denselben Bedingungen ab. Verglichen mit der liegenden Position kam es bei beiden Altersgruppen in beiden Seitenlagen zu einer signifikanten Umverteilung der regionalen Ventilation. Eine stärkere Beteiligung an der Gesamtventilation lässt sich für die jeweils oben liegende Lunge beobachten. Dieses Verhalten kehrte sich während der letzten Phase der Messung, also der Spontanatmung nahe der Totalkapazität um (Abbildung 17, unten). Bei hohem Lungenvolumen nahe der TLC war die Ventilation in der jeweils unten liegenden Lunge deutlich höher als die Ventilation in der oben liegenden Lunge. Dieser Sachverhalt ist in beiden Altersgruppen zu erkennen. 


\section{Diskussion}

\subsection{Diskussion der Methodik}

Die Überwachung der Atmung unter klinischen Bedingungen erfolgt häufig mit Methoden, die atemsynchrone Widerstandsänderungen auf der Körperoberfläche erfassen. Dieser Trend hat sich aus der kardiologischen Überwachung abgeleitet. Es zeigte sich, dass Änderungen des pulmonalen Luftgehaltes ebenfalls elektrische Widerstandsveränderungen verursachen, die methodisch für die Fragestellung der pulmonalen Gasverteilung genutzt werden können (Harris et al. 1987, Adler et al. 1997). Erste Untersuchungen an beatmeten Hunden haben nachgewiesen, dass pulmonale Volumenveränderungen zuverlässig erfasst werden können, allerdings mit geringer räumlicher Auflösung (Adler et al. 1997).

Die Methode wurde inzwischen weiterentwickelt. Das Ziel soll ein Verfahren sein, dass bettseitig einsetzbar ist und in kurzer Zeit relevante regionale pulmonale Daten bereitstellen kann. Die bettseitige Einsetzbarkeit ist heute bereits gegeben, der Rechenaufwand ist aber noch zu groß, um für klinische Fragestellungen der Intensivmedizin unmittelbar Anwendung $\mathrm{zu}$ finden. Es handelt sich um eine strahlungsfreie Datenerfassung mit exzellenter zeitlicher Auflösung. Das kleinste Lungenvolumen, in dem eine regionale Änderung der Gasverteilung erfasst werden kann, liegt gegenwärtig bei $25 \mathrm{ml}$ (Hahn et al. 2002). Der Einsatz von Oberflächenelektroden kennzeichnet ein geringes $\mathrm{Maß}$ an Invasivität. Die Oberflächenelektroden sind in unterschiedlicher Höhe einsetzbar, je nach der Fragestellung der Untersuchungen. Die Validität der Methode wurde mit experimentellen und klinischen Methoden bestätigt. Dies gilt für die Spirometrie (Harris et al. 1987, Adler et al. 1997), Auswaschtechniken mit Inertgasen (Hinz et al. 2003a), Ventilationsszintigraphie (Serrano et al. 2002), Single-Photonen Emission (Hinz et al. 2003b) sowie Elektronenstrahl-CT (Frerichs et al. 2002).

Intrathorakale Blutvolumenveränderungen erzeugen ebenfalls thorakale Impedanzveränderungen, allerdings ist der Effekt sich ändernder Blutvolumina deutlich geringer als bei den Luftveränderungen. Die größten Änderungen des Blutvolumens erfolgen im Bereich der Herzens und der großen Gefäße. In der vorliegenden Studie wurden diese Regionen nicht in die Analyse mit einbezogen. 
Die Fluktuationen des Blutvolumens im Lungengewebe dürften die Ergebnisse nur geringfügig beeinflusst haben, ebenso wie atemsynchrone Blutvolumenveränderungen.

Inzwischen ist die EIT recht robust und ermöglicht eine unbehinderte Datenerfassung auch in einer elektrisch kontaminierten Umgebung wie auf Intensivstationen. Die Nützlichkeit des Verfahrens für klinische Entscheidungen sollte in der Zukunft durch weitere Untersuchungen an Patienten belegt werden. Die Mehrzahl der Intensivpatienten dürfte einer älteren Altersklasse angehören. Es war daher unsere Absicht, ältere Individuen unter physiolgischen Bedingungen zu untersuchen, um das altersbedingte topographische Muster der Gasverteilung zu erfassen.

Unsere gegenwärtigen Kenntnisse der topographischen Gasverteilung in der Lungen wurde durch Studien mit inhalierten radioaktiven Spurengasen, z.B. ${ }^{133} \mathrm{Xe}$ und externen Strahlungsmessgeräten gewonnen. Dazu gehören insbesondere die Arbeiten von Milic-Emili et al. (1966) und Holland et al. (1968). Diese Arbeiten beziehen sich auf die räumliche Gasverteilung bei gesunden aufrechten jungen und älteren Individuen, die mit verschiedenen Atemmanövern erfasst wurde. Diese experimentellen Daten erlauben einige Rückschlüsse über Gasverteilungskriterien in beiden Altersklassen. Einige von unseren Untersuchungsergebnissen befinden sich in Übereinstimmung mit diesen frühen szintigraphischen Studien.

\subsection{Diskussion der Ergebnisse}

In dieser Studie wurden die Effekte des Alterns auf die regionale Ventilation in verschiedenen Körperlagen und während verschiedener Atemmanövern untersucht. Das bedeutet, dass die Ergebnisse dieser Studie als Effekte einer Kombination von Alterung und 1. Körperlage (sitzend, liegend, rechte und linke Seitenlage), 2. Art der Atmung (Spontanatmung oder tiefe Atmung), 3. Gasfluss der Atmung (schnell oder langsam) und 4. Lungenvolumen interpretiert werden müssen. Im Folgenden werden die Ergebnisse diskutiert, bei denen sich ein signifikanter Effekt der Alterung auf die intrapulmonale Gasverteilung darstellt. 


\subsubsection{Effekte von Alter und Körperlage}

Bei den alten Probanden zeigte sich während normaler Spontanatmung keine lageabhängige Umverteilung der Ventilation (Abbildung 13 und Abbildung 17, oben). Dieses Verhalten lässt sich mit früherem Atemwegsverschluss (airway closure) in den jeweils unten liegenden Anteilen der Lunge bei älteren Menschen erklären (Leblanc et al. 1970, Rehder 1998). Während der Atemmanöver mit vollständiger Exspiration (VC und FVC) bei den älteren Probanden kam es in der vermessenen Ebene bei der Lageänderung von sitzender in liegende Position zu einer Abnahme des Anteils, den die rechte Lunge an der Gesamtventilation einnimmt (Abbildung 13).

\subsubsection{Effekte von Alter und Art der Atmung}

Während der vollständigen Ausatmung haben sich bei den alten Probanden deutlich geringere Änderungen des regionalen Gasvolumens gezeigt (Abbildung 12), was der Ausatmung kleinerer regionaler Volumina zwischen der TLC und dem RV entspricht. Diese Ergebnisse stimmen logisch mit den durch die Lungenfunktion erhobenen Befunden überein, die eine verminderte VC und ein erhöhtes RV in der Gruppe der alten Probanden zeigen.

\subsubsection{Effekte von Alter und Atemgasfluss}

Hoher Gasfluss vermindert die räumliche Inhomogenität der Ventilation (Jones und Clarke 1969, Millette et al. 1969) erhöht bei alten Menschen durch eine verminderte mechanische Stabilität des Lungengewebes allerdings die Anfälligkeit der Atemwege zu kollabieren (Edelman et al. 1968, Gibson et al. 1976). Im Einklang mit diesen Erkenntnissen zeigte sich eine stärkere Ventilation der unten liegenden Lunge bei der langsamen Exspiration (Abbildung 13, unten). Bei der forcierten Exspiration ist der Anteil, den die unten liegende Lungenregion einnimmt, reduziert (Abbildung 13, mittig). Der Vergleich der Volumenänderung, die zwischen der totalen Lungenkapazität und der funktionellen Residualkapazität auftritt, mit der Volumenänderung bei langsamer (VC) und forcierter (FVC) Exspiration (Abbildung 15), zeigt höhere relative Werte in der Gruppe der alten Probanden. 
Dies spricht für früheren Atemwegsverschluss (airway closure) bei den alten Probanden. Der frühere Verschluss von Atemwegen während der Atmung mit hohen Flussraten bei den alten Probanden bestätigt sich durch die Ausatmung größerer regionaler Volumina bei der langsamen Ausatmung verglichen mit der forcierten Entleerung der Lunge (Abbildung 12). Am deutlichsten wurde dieser Unterschied in den jeweils unten liegenden Lungenabschnitten in den Seitenlagen.

\subsubsection{Effekte von Alter und Lungenvolumen}

Während der Spontanatmung mit einem geringen Lungenvolumen (Abbildung 17, mittig) nahm in der vermessenen Ebene bei einem Lagewechsel von der sitzenden in die liegende Position der Anteil, den die rechte Lunge an der Gesamtventilation einnimmt, bei den jungen Probanden zu, während er bei den alten Probanden abnahm. Diese Ergebnisse ergeben sich aus einem Abfall der regionalen Ventilation, die bei den jungen Probanden vorwiegend die linke Lunge betrifft und bei den alten vorwiegend die rechte Lunge. Eine mögliche Erklärung für diesen Unterschied zwischen jungen und alten Probanden ist eine unterschiedlich stark ausgeprägte Zwerchfellbewegung, die sich durch eine unterschiedliche Verlagerung der intraabdominaler Organe ergibt. In jüngerem Alter ist die Kuppel der rechten Zwerchfellseite stärker gewölbt als die linke Seite und somit ist auch die Volumenverdrängung der rechten Zwerchfellseite im Liegen größer (Krayer et al. 1989). Ein reduzierter Gasfluss in die rechten Lungenregionen zeigte sich bei den alten Probanden nur während der Atmung mit einem geringen Gesamtlungenvolumen. Es kann davon ausgegangen werden, dass dieses Atemmuster durch eine insgesamt reduzierte Beweglichkeit des Brustkorbes und eine geringere Elastizität der Lunge entsteht, die zu einer Abflachung der Zwerchfellkuppel führt. Dadurch entsteht ein ungünstigeres Verhältnis zwischen Länge und Spannung der Muskelfasern. Dieser Mechanismus greift allerdings nur, wenn die Dehnbarkeit des Zwerchfells einheitlich ist (Rehder 1998). Es ist jedoch nicht bekannt, ob Spannung und Dehnbarkeit des Zwerchfells im Alter gleichmäßig verteilt sind. Im allgemeinen können die Unterschiede in der regionalen Ventilation zwischen den jungen und alten Probanden durch Veränderungen der Atemmechanik erklärt werden, die durch den Alterungsprozess hervorgerufen werden (Gibson et al. 1976, Rossi et al. 1996, Babb und Rodarte 2000, Zeleznik 2003). 
Diese funktionelle und strukturelle Lungenalterung ist für die globalen Veränderungen der Ventilation und Lungenvolumnina wie beispielsweise der Zunahme des Residualvoumes (RV) und der funktionellen Residualkapazität (FRC), sowie der Abnahme der Vitalkapazität (VC), der forcierten Vitalkapazität (FVC) und der Einsekundenkapazität $\left(\mathrm{FEV}_{1}\right)$ verantwortlich und wird bei den alten Probanden auch durch die Lungenfunktionsuntersuchung deutlich.

In dieser Studie wurden die Änderungen des regionalen Lungenvolumens in einer kaudal gelegenen Lungenregion gemessen, in der ein komplexes Zusammenspiel zwischen Brustkorbbewegungen, dem Zwerchfell, der intraabdominalen Organe und der mediastinalen Organe und Strukturen die regionale Ventilation beeinflussen. Viele einzelne Komponenten dieses komplexen Systems werden durch den Alterungsprozess beeinflusst und die Ergebnisse dieser Studie zeigen, dass deutliche Unterschiede in der regionalen Ventilation zwischen jungen und alten Menschen bestehen. Obwohl einige Effekte des Alterns auf die Ventilation bekannt sind, wie zum Beispiel früher Atemwegsverschluss bei der Ausatmung, das schon im Bereich der normalen Spontanatmung zum Tragen kommt (Holland et al. 1968; Leblanc et al. 1970), oder die Zunahmen von Atemwegsverschlüssen in liegender Position (Leblanc et al. 1970), ist es schwierig, einzuschätzen, welche Faktoren in welcher Ausprägung relevant werden und in welchem Ausmaß die regionale Ventilation beeinflusst wird. Um genauere Aussagen über den Einfluss der sich im Alter verändernden einzelnen Komponenten machen zu können und um genau beschreiben zu können, wie sich die einzelnen Faktoren in dem komplexen System von Brustkorb und Lunge auf die regionale Ventilation auswirken, wären eine Vielzahl von weiteren Untersuchungen notwendig. So wäre während der verschiedenen Atemmavöver eine durchgehende Messung des intrapleuralen Drucks notwendig. Außerdem müssten mit bildgebenden Verfahren genaue Kenntnisse über die räumlichen Veränderungen des Brustkorbes, exakte morphologische Gegebenheiten des Zwerchfells und seiner Bewegungen, sowie detaillierte anatomische Kenntnisse über die Lage und Größe der intraabdominellen und mediastinalen Organe erhoben werden. Detaillierte Daten, die sich mit den genauen Prozessen der durch die Alterung bedingten Veränderungen der Ventilation befassen, sind bis zum heutigen Tag aus ethischen und methodischen Gründen nicht verfügbar. 


\subsection{Möglichkeiten der klinischen Anwendung}

Die Erfassung von Störungen der Ventilation und Gasverteilung mit raschen Atemgasanalysen und Ein- und Auswaschverfahren mittels Helium, Stickstoff und radioaktivem Xenon hat die Kenntnisse der Gasaustauschvorgänge ganz wesentlich erweitert. Dies bezieht sich sowohl auf physiologische als auch auf pathophysiologische Vorgänge. West (West 1970) hat diese Zusammenhänge in seiner Monographie "Ventilation/blood flow and gas exchange" übersichtlich dargestellt. Bei der alveolo-arteriellen Differenz für Atemgase und analog auf der Blutseite bei der venösen Beimischung der Lunge zum arteriellen Blut finden sich Fraktionen dieser Differenzen, die auch beim Gesunden auf regionale Gasverteilungsvorgänge zurückzuführen sind. Pathophysiologische Zustände wie bei $\mathrm{CO}_{2}$-Retention verstärken diesen Einfluss, wobei regionale Veränderungen und die Schwerkraft eine Rolle spielen. Dabei kann der Einfluss von Inhomogenitäten der Gasverteilung sowohl während des ganzen Atemzyklus wirksam sein als auch nur teilweise, wenn sich eine pulmonale Region nach der Ausatmung erst im Verlaufe der Inspiration öffnet. Die Vorstellungen über den Einfluss von ventilatorischen Verteilungsstörungen haben sich aus zahlreichen verschiedenen Einzelanalysen und Untersuchungsserien ergeben.

Welche Möglichkeiten des wissenschaftlichen und klinischen Einsatzes kann nun eine Methode wie die EIT bieten, die mobil einsetzbar ist, eine gute räumliche und sehr gute zeitliche Auflösung bietet und ohne Invasivität kontinuierlich einsetzbar ist?

Es gibt einen dringenden Bedarf für Untersuchungsverfahren, die die regionale Ventilation und Gasverteilung direkt am Krankenbett erfassen können. Pulmonale Krankeitsprozesse, die eine Indikation zur apparativen Beatmung darstellen (Frerichs et al. 2001a, Rimensberger 2002), sind meist durch regionale Veränderungen geprägt. Die konventionellen Untersuchungsverfahren der Intensivmedizin sind bezüglich der Aussage zur regionalen Ventilation bzw. Gasverteilung limitiert (Caples und Hubmayr 2003). 
Dabei haben mehrere klinische und experimentelle Valdierungsstudien gezeigt, dass eine EIT-Untersuchung Änderungen des regionalen Luftgehaltes z.B. im Vergleich zu Computertomographie (Frerichs et al. 2002), Ventilationsszintigraphie (Serrano et al. 2002) und SPECT (single-photon-emission computed tomography, nuklearmedizinisches diagnostisches Verfahren mit Erzeugung von Schnittbildern nach Injektion, Verteilung und Organanreicherung von Radiopharmaka, (Hinz et al. 2003b) richtig wiedergibt. Moderne Beatmungsstrategien der Intensivmedizin tendieren zur Invasivität, wenn die pulmonale Krankheit fortschreitet. Veränderungen des Inspirations-/Exspirations-Verhältnisses und eine Zunahme des Beatmungsdruckes (PEEP: positive endexpiratory pressure) können den Gasaustausch, erkennbar an der alveolo-arteriellen Sauersoffdruck-Differenz verbessern. Neben anderen unerwünschten Effekten vermindert der positive intrathorakale Druckverlauf jedoch den Blutrückstrom zum Herzen mit Erniedrigung des Herz-Zeit-Volumens. Mit der EIT-Methode ist unter diesen Umständen eine PEEP-Titration möglich, bei der in Kombination mit den konventionellen Überwachungsverfahren der Intensivmedizin die Qualität des Gasaustausches und der Gesamtzirkulation, ergänzt durch die Erfassung der regionalen Ventilation und Gasverteilung möglich ist. Gleiches gilt für die Anwendung von alveolären Recruitment-Manövern mit der kurzzeitigen Anwendung von hohen Beatmungsdrucken zur Wiedergewinnung verloren gegangener Alveolarbereiche. Der direkte kontinuierlich abrufbare diagnostisch-therapeutische Zugang zur regionalen Ventilation und Gasverteilung stellt für die Beatmungsstrategie einen großen Fortschritt dar, mit dem erstmals die regionale Effektivität von Beatmungsmaßnahmen direkt überprüfbar werden kann.

Die beschriebene EIT-Anwendung zur Optimierung von Beatmungsstrategien kann aktuell noch nicht in der beschriebenen Weise eingesetzt werden, da eine Software zur Datenauswertung in Echtzeit noch nicht verfügbar ist. Darüber hinaus wäre es wünschenswert, wenn die weitere Entwicklung des EIT-Verfahrens in Richtung auf die Nutzung mehrerer in verschiedener Höhe abrufbarer Schnittbilder geführt werden könnte. Dies wäre der Einstieg in eine dreidimensionale Betrachtung der regionalen Ventilation und Gasverteilung. 


\section{Zusammenfassung}

Die Gasverteilung ist neben der Ventilation, Diffusion und Lungenperfusion ein wesentlicher Teilprozess der Lungenfunktion. Bei der gesunden Lunge gibt es eine relativ homogene Gasverteilung, bei der sehr viele Alveolen gleich schnell belüftet werden. Chronische Krankheitsprozesse der Lunge zeichnen sich dadurch aus, dass viele Lungenbläschen unterschiedlich schnell ventiliert werden und einzelne Teilbereiche der Lunge während maximaler Atemmanöver und auch normaler Ruheatmung verschlossen sind. Dabei erscheint die Lunge als ein Organ, bei dem die Gasverteilung im Zusammenhang mit der Ruhedehnungskurve und ihrem Eigengewicht vorwiegend in den basalen Bereichen abläuft. Damit ist die Gasverteilung ein regionaler Prozess. Der Alterungsprozess verschlechtert wie chronische Krankheitsprozesse die Qualität der Gasverteilung, nur nicht so ausgeprägt. Die mit Fremdgas- und Stickstoffauswaschverfahren ermittelten Untersuchungsergebnisse liefern zwar einen globalen Hinweis auf die Qualität der Gasverteilung, können jedoch keine Auskunft darüber geben, wo bei der Ruheamtung und bei verschiedenen Atemmanövern die Veränderungen der Gasverteilung auftreten.

Dies kann eine neuartige Methode, die EIT, die bettseitig einsetzbar ist und ohne Strahlenbelastung thorakale Schnittbilder erzeugt. Bei dieser Methode werden über 16 Oberflächenelektroden, die in einer Ebene symmetrisch über den Brustkorb verteilt sind, nach einer Einspeisung eines schwachen Wechselstroms die resultierenden Spannungen gemessen. Die Widerstandsänderungen im Brustkorb sind primär von der durch die Ventilation bzw. die nachgeschaltete Gasverteilung bewegten Luftmenge abhängig. Durch aufwendige Verfahren der Bildrekonstruktion kann aus den gemessenen Spannungsdifferenzen auf der Körperoberfläche ein transversales Schnittbild des Thorax erzeugt werden, das funktionell Informationen über die regionale Gasverteilung liefert. Die Methode hat ein brauchbares räumliches und ein sehr gutes zeitliches Auflösungsvermögen. Sie steht noch nicht als kommerzielles Verfahren zur Verfügung, kann jedoch bereits für grundsätzliche Fragestellungen eingesetzt werden. Eine solche in dieser Arbeit verfolgte Fragestellung sind die altersbedingten Veränderungen der Gasverteilung bei gesunden Probanden verschiedener Altersklassen, insbesondere bei Lageveränderungen und verschiedenen Atemmanövern. 
Die Fragestellung ergibt sich daraus, dass viele Patienten in der Intensivmedizin, für die die Methode von Interesse ist, einer höheren Altersklasse angehören. Damit können Veränderungen der Gasverteilung in höherem Lebensalter prinzipiell dem Krankeitsprozess, aber auch dem Lebensalter zugeordnet werden.

Es wurden insgesamt 16 gesunde Probanden, davon 8 junge und 8 ältere mit einem Durchschnittsalter von 26 bzw. 75 Jahren, in die Studie mit einbezogen. Die Studie wurde von der zuständigen Ethikkommission der Medizinischen Fakultät genehmigt. Die Atemmanöver "normale Ruheatmung“, „schnelle und langsame maximale Ausatmung" sowie zusätzlich "Ruheatmung in verschiedenen Atemstellungen“ wurden bei allen Probanden durchgeführt. Zusätzlich erfolgte die Erhebung der konventionellen spirometrischen und bodyplethysmographischen Funktionsdaten mit dem Lungenfunktionsgerät Masterlab der Fa. Jäger, Würzburg.

Die Lungenfunktionsuntersuchungen ergaben Normalwerte, mit diskreten pathologischen Abweichungen für je einen Probanden in beiden Altersklassen.

Bei der Ruheatmung zeigte sich bei den jüngeren im Gegensatz zu den älteren Probanden eine signifikante Redistribution der Gasverteilung zugunsten der unteren Lunge. Bei der Bestimmung des relativen Anteils der rechten Lungen an der Gesamtventilation bei voller Ausatmung nach tiefer Inspiration ergab sich dementsprechend bei den jungen Probanden eine hochsignifikante Gasumverteilung zugunsten der unteren Lunge bei Lagewechsel in die rechte oder linke Seitenlage. In beiden Altersgruppen waren die regionalen FVC- und VC-Werte, die als Summe der relativen endinspiratorischen und endexspiratorischen Spannungsänderungen erfasst wurden, signifikant höher als die korrespondierenden Werte bei Ruheatmung. Dabei waren diese FVC- und VC-Werte bei den jüngeren für alle Positionen signikant höher als bei den älteren Probanden.

Der Vergleich des örtlichen Lungenvolumens nach den FVC- und VC-Manövern im Vergleich zur Atemmittellage bei FRC zeigte, dass dieses Verhältnis bei den älteren durchweg höher war als bei den jüngeren Studienteilnehmern. Dies entspricht einer stärker eingeschränkten regionalen Luftentleerung im höheren Lebensalter. Dabei konnte auch nachgewiesen werden, dass die nicht-abhängige Lungenpartie einen Luftverlust bis deutlich unter das FRC-Niveau erleidet, während die Entleerung der abhängigen Lunge viel näher am regionalen FRC-Niveau endet, etwas darunter bei den jüngeren, darüber bei den älteren Probanden. 
Bei der Ruheatmung auf verschiedenen Niveaus der Lungenfüllung wurde bei beiden Altersklassen eine signifikante Umverteilung der Atemgase für jede Seitenposition im Verhältnis zum Liegen beobachtet. Bei Ruheatmung auf RV-Niveau war die Belüftung der nicht-abhängigen Partie stärker ausgeprägt als die der anderen Seite. Dieses Verteilungsmuster der Atemgase wurde bei Atmung auf TLC-Niveau umgedreht, so dass der Gasfluss in die abhängige Lungenpartie viel ausgeprägter war.

Bei einer zusammenfassenden Bewertung der Ergebnisse wird das Konzept einer schwerkraftabhängigen, an der Ruhedehnungskurve der Lunge orientierten regionalen Gasverteilung mit Verschluss der abhängigen Lungenpartien bestätigt. Im Alter erreicht dieser Prozess durch den Elastizitätsverlust und die Änderungen der Atemmechanik auch die normale Ruheatmung (Abbildungen 12,13,14,15,17). Die Zunahme des Anteils des RV an der TLC ist ebenfalls auf diesen funktionellen und strukturellen Prozess zurückzuführen (Tabelle 2). Durch die Ruheatmung auf dem Niveau der TLC, also mit gefüllter Lunge, wird dieser Mechanismus unabhängig vom Alter scheinbar vollständig aufgehoben (Abbildung 7 unten). Die Ruheatmung der älteren Probanden auf FRC-Niveau wird derjenigen der jungen, die auf RV-Niveau atmen, ähnlich (Abbildung 7, oben, rechts, links und mittig).

Die EIT-Methode hat sich mit einer bereits im gegenwärtigen Entwicklungsstand gut brauchbaren räumlichen und exzellenten zeitlichen Auflösung bewährt. Die rasche zeitliche Erfassung von Änderungen der regionalen Gasverteilung erkennt man besonders in Abbildung 5, bei der nach dem schnellen FVC-Manöver das Ausmaß der regionalen Gasretention im Alter erkennbar wird. Damit ist es erstmals möglich, mit einem auf das Lungengewebe gerichteten, wenig invasiven Verfahren rasche Änderungen der regionalen Gasverteilung zu erfassen. Dies wäre besonders interessant im Bereich maschineller Beatmung auf der Intensivstation. Hier können Informationen zur Qualität der Gasversorgung zusätzliche diagnostische und therapeutische Hinweise geben. 


\section{Literaturverzeichnis}

Adler A, Amyot R, Guardo R, Bates JHT, Berthiaume Y (1997): Monitoring changes in lung air und liquid volumes with electrical impedance tomography. J Appl Physiol $\underline{83}, 1762-1767$

Babb TG, Rodarte JR (2000): Mechanism of reduced maximal exspiratory flow with aging. J Appl Physiol $\underline{89}$, 505-511

Bates DV, Macklem PT, Christie RV; Respiratory function in disease; Second edition; WB Saunders Company, Philadelphia, London, Toronto 1971, S 34 und 43-47

Braun U (1988): A.L. Lavoisier und der Anaesthesist. Anaesthesist $\underline{37}, 664-671$

Braun U, Voigt E (1978): Die Rolle von ventilatorischen Verteilungsstörungen bei der späten postoperativen Hypoxämie nach Oberbauchlaparotomien. Anaesthesist $\underline{27}$, 163-171

Brown DC, Barber BH (1989): A review of image reconstruction techniques for electrical impedance tomography. Med Phys $\underline{16}, 162-169$

Campbell EJ, Lefrak SS (1978): How aging affects the structure and function of the respiratory system. Geriatrics $\underline{33}, 68-74$

Caples SM, Hubmayr RD (2003): Respiratory monitoring tools in the intensive care unit. Curr Opin Crit Care $\underline{9}, 230-235$

Comroe JH, Fowler WS (1951): Lung function studies. VI: Detection of uneven alveolar ventilation during a single breath of oxygen; a new test of pulmonary disease. Am J Med 므, 408

Edelman NH, Mittman C, Norris AH, Shock NW (1968): Effects of respiratory pattern on age differences in ventilation uniformity. J Appl Physiol 24, 49-53 
Fleisch A (1925): Der Pneumotachograph; ein Apparat zur Geschwindigkeitsregistrierung der Atemluft. Pflügers Arch Ges Physiol 209, 713

Frerichs I, Dudykevych T, Hinz J, Bodenstein M, Hahn G, Hellige G (2001a): Gravity effects on regional lung ventilation determined by functional EIT during parabolic flights. J Appl Physiol 91, 39-50

Frerichs I, Schiffmann H, Hahn G, Hellige G (2001b): Noninvasive radiation-free monitoring of regional lung ventilation in critically ill infants. Intensive Care Med $\underline{27}$, 1385-1394

Frerichs I, Hinz J, Herrmann P, Weisser G, Hahn G, Dudykevych T, Quintel M, Hellige G (2002): Detection of local lung air content by eletrical impedance tomograpy compared with electron beam CT. J Appl Physiol $\underline{93}, 660-666$

Geddes LA, Baker LE (1967): The specific resistance of biological materials - a compendium of data for the biomedical engineer and physiologist. Med Biol Eng $\underline{5}$, 271-293

Gibson GJ, Pride NB, O'Cain C, Quagliato R (1976): Sex and age differences in pulmonary mechanics in normal nonsmoking subjects. J Appl Physiol 41, 20-25

Hahn G, Hartung C, Hellige G: Elektrische Impedanztomographie (EIT) als Methode zur regionalen Beurteilung der Lungenventilation. Akademie der Wissenschaften und der Literatur, Mainz, Germany, Mainz 1996

Hahn G, Dudykevych T, Frerichs I, Thiel F, Hellige G: A high performance electrical impedance tomography (EIT) system for clinical evaluation studies and space application. In: Hutten H, Krösl P, (Eds.), Proceedings of the Second European Medical and Biological Engineering Conference. Verlag der TU Graz, Graz 2002, $110-111$ 
Harris ND, Suggett AJ, Barber DC, Brown BH (1987): Applications of applied potential tomography (APT), Respiratory Medicine. Clin Phys Physiol Meas $\underline{8}$ (Suppl. A), 155-165

Hinz J. Hahn G, Neumann P, Sydow M, Mohrenweiser P, Hellige G, Burchardi H (2003a): End-expiratory lung impedance change enables bedside monitoring of endexpiratory lung volume change. Intensive Care Med 29, 37-43

Hinz J, Neumann P, Dudykevych T, Andersson LG, Wrigge H, Burchardi H, Hedenstierna G (2003b): Regional ventilation by electrical impedance tomography: a comparison with ventilation scintigraphy in pigs. Chest $\underline{124}, 314-322$

Holland J, Milic-Emili J, Macklem PT, Bates DV (1968): Regional distribution of ventilation and perfusion in elderly subjects. J Clin Invest $\underline{47}, 81-92$

Jones JG, Clarke SW (1969): The effect of exspiratory flow rate on regional lung emptying. Clin Sci $\underline{37}, 343-356$

Krayer S, Rehder K, Vettermann J, Didier P, Ritman EL (1989): Position and motion of the human diaphragm during anaesthesia-paralysis. Anesthesiology $\underline{70}, 891-898$

Kronenberg RS, Drage CW, Ponto RA, Williams LE (1973): The effect of age on the distribution of ventialtion and perfusion in the lung. Am Rev Respir Dis $\underline{108}, 576-586$

Leblanc P, Ruff F, Milic-Emili J (1970): Effects of age and body position on "airway closure" in man. J Appl Physiol $\underline{28}, 448-451$

Lyons AS, Keiner M, Lyons AS, Petrocelli II (Eds.): Die Geschichte der Medizin im Spiegel der Kunst, DuMont Buchverlag Köln, Köln 1980, 433

Milic-Emili J, Henderson JAM, Dolovich MB, Trop D, Kaneko K (1966): Regional distribution of inspired gas in the lung. J Appl Physiol 21, 749-759 
Millette B, Robertson PC, Ross WRD, Anthonisen NR (1969): Effect of exspiratory flow rate on emptying of lung regions. J Appl Physiol $\underline{27}, 587-591$

Quanjer PH, Tammeling GJ, Cotes JE, Pederson OF, Peslin R, Yernault JC (1993): Lung volumes and forced ventilatory flows. Report working party standardization of lung function tests, European Community for steel and coal. Official statement of the European Respiratory Society. Eur Resp J $\underline{6}$ (Suppl. 16), 5-40

Rehder K (1998): Postural changes in respiratory function. Acta Anaesthesiol Scand $\underline{42}$ (Suppl. 113), 13-16

Rimensberger PC (2002): Neonatal respiratory failure. Curr Opin Pediatr 14, 315-321

Rossi A, Ganassini A, Tantucci C, Grassi V (1996): Aging and the respiratory system. Aging $\underline{8}, 143-161$

Seguin A, Lavoisier AL (1783): Premier mémoire sur la respiration des animaux. Mém acad R Sci, 566. Oeuvres, Vol II, Dumas-Grimaux, Paris, 1862-1893, 688

Serrano RE, de Lema B, Casas O, Feixas T, Calaf N, Camacho V, Carrio I, Casan P, Sanchis J, Riu PJ (2002): Use of electrical impedance tomography (EIT) for the assessment of unilateral pulmonary function. Physiol Meas $\underline{23}, 211-220$

Stenqvist O (2003): Practical assessment of respiratory mechanics. Br J Anaesth $\underline{91}$, 92-105

Ulmer WT, Reichel G, Nolte D, Islam MS: Die Lungenfunktion. Physiologie und Pathophysiologie, Methodik. 5. Auflage; Georg Thieme Verlag, Stuttgart 1991

West JB: Ventilation/blood flow and gas exchange. Second edition, Blackwell Scientific Publications, Oxford, London, 1970

Zeleznik J (2003): Normative aging of the respiratory system. Clin Geriatr Med $\underline{19}$, $1-18$ 